\title{
Change-point estimation in $\mathrm{ARCH}$ models
}

\author{
PIOTR KOKOSZKA ${ }^{1}$ and REMIGIJUS LEIPUS ${ }^{2}$ \\ ${ }^{1}$ Department of Mathematical Sciences, University of Liverpool, Liverpool L69 3BX, UK. \\ E-mail: p.s.kokoszka@liv.ac.uk \\ ${ }^{2}$ Department of Mathematics, Vilnius University, Naugarduko 24, Vilnius 2600, Lithuania. \\ E-mail: remigijus.leipus@maf.vu.lt
}

This paper studies the change-point problem and the cross-covariance function for ARCH models. Bounds for the cross-covariance function are derived and explicit formulae are obtained in special cases. Consistency of a CUSUM type change-point estimator is proved and its rate of convergence is established. A Hájek-Rényi type inequality is also proved. Results are obtained under weak moment assumptions.

Keywords: ARCH model; change-point estimation; cross-covariance function

\section{Introduction}

This paper studies ARCH models defined by the equations

$$
r_{k}=\sigma_{k} \varepsilon_{k}, \quad \sigma_{k}^{2}=a+\sum_{j=1}^{\infty} b(j) r_{k-j}^{2},
$$

where the $\varepsilon_{k}$ are independent and identically distributed (i.i.d.) errors and $a$ and the $b(j)$ are non-negative constants. Since their introduction by Engle (1982), ARCH type models have become perhaps the most popular and extensively studied financial econometric models. The literature on the subject is so vast that we will restrict ourselves to directing the reader to fairly comprehensive reviews by Bollerslev et al. (1992) and Shephard (1996). Model (1.1) is suitable for series $r_{k}$ such that the observations are uncorrelated but exhibit 'clusters of volatility'.

We study the estimation of a change-point in (1.1). Note first that if the $\varepsilon_{k}$ have mean zero and unit variance and the sequence $\left\{r_{k}\right\}$ is weakly stationary, then

$$
\operatorname{var}\left[r_{k}\right]=a /\left(1-\sum_{j=1}^{\infty} b(j)\right) .
$$

Suppose now that the parameters $a$ and $b(j)$ change at an unknown point $k^{*}$ in such a way that the variance given by (1.2) changes. We are interested in estimating the change-point $k^{*}$. This problem is different from the problem of testing for structural changes where $k^{*}$ is assumed to be known and one tests whether a change in model structure has taken place at $k^{*}$. Hsu (1977), Inclán and Tiao (1994) and Chen and Gupta (1997) studied a simpler 
variance-change model $r_{k}=\tilde{\sigma}_{k} \varepsilon_{k}$, where $\tilde{\sigma}_{k}^{2}=a_{1}$, if $1 \leqslant k \leqslant k^{*}$, and $\tilde{\sigma}_{k}^{2}=a_{2}$, if $k^{*}<k \leqslant n$, and where $\left\{\varepsilon_{k}\right\}$ is a sequence of independent standard normal random variables. Horváth (1993) considered tests against alternatives allowing changes both in means and variances of a sequence of independent normal random variables, whereas estimators and tests for change in variance under weak moment conditions were considered in Gombay et al. (1996).

By contrast, the change-point problem for linear sequences has received a great deal of attention. Csörgö and Horváth (1988; 1997) and Brodsky and Darkhovsky (1993) focus mainly on sequences of independent observations. Recently there has, however, been increasing interest in dependent data; see Antoch et al. (1997), Bai (1994), Bai and Perron (1998), Davis et al. (1995), Giraitis et al. (1996), Giraitis and Leipus (1990; 1992), Horváth and Kokoszka (1997) and Leipus (1994), among others. The present paper studies processes which exhibit no linear dependence, but are characterized by the dependence of the conditional variance on past observations. This is known in econometrics as 'dependence in volatility'.

We study a CUSUM type estimator $\hat{k}$ of $k^{*}$ defined by

$$
\hat{k}=\min \left\{k:\left|R_{k}\right|=\max _{1 \leqslant j \leqslant n}\left|R_{j}\right|\right\}
$$

where

$$
R_{k}=\frac{k(n-k)}{n^{2}}\left(\frac{1}{k} \sum_{j=1}^{k} r_{j}^{2}-\frac{1}{n-k} \sum_{j=k+1}^{n} r_{j}^{2}\right)
$$

Figure 1 illustrates how the method works. Figure 1(a) shows a simulated realization $\left\{r_{k}, 1 \leqslant k \leqslant 1000\right\}$ of an $\mathrm{ARCH}(1)$ sequence with conditional variance $a_{1}+b r_{k-1}^{2}$ for $k \leqslant 700$ and $a_{2}+b r_{k-1}^{2}$ for $k>700$. We set $a_{1}=1, a_{2}=1.3, b=0.1$ and used standard normal innovations $\varepsilon_{k}$. It is impossible to tell by eye if and where a change in variance occurred. Figure 1(b) shows the corresponding sequence $R_{k}$. The maximum is attained at $\hat{k}=699$.

Despite the standard form of the estimator $\hat{k}$, proving its consistency is not easy. For analogous estimators of a change-point in the mean of a linear process $X_{k}=\mu_{k}+e_{k}$ the dependence structure of the errors $e_{k}$ must be known. For ARCH models, we must be able to say something about the cross-covariance function of two $\mathrm{ARCH}$ sequences driven by the same innovations $\varepsilon_{k}$. This issue, which takes up the bulk of the paper, is addressed in Section 3. We believe that the results obtained in Section 3 are also of independent interest, as the study of the covariance function of ARCH type sequences has occupied an important place in their theory. The method we propose is based on a Volterra series type expansion of the random variables $r_{k}^{2}$ derived in Section 2. It is different from methods based on matrix manipulations, which go back to Engle (1982) and are applicable only to finite-order ARCH and GARCH models; see He and Teräsvirta (1997) and references therein.

Another difficulty lies in the complex probabilistic structure of the sequence $\left\{r_{k}^{2}\right\}$, which does not fall into any well-studied class of stochastic processes for which appropriate 
(a)

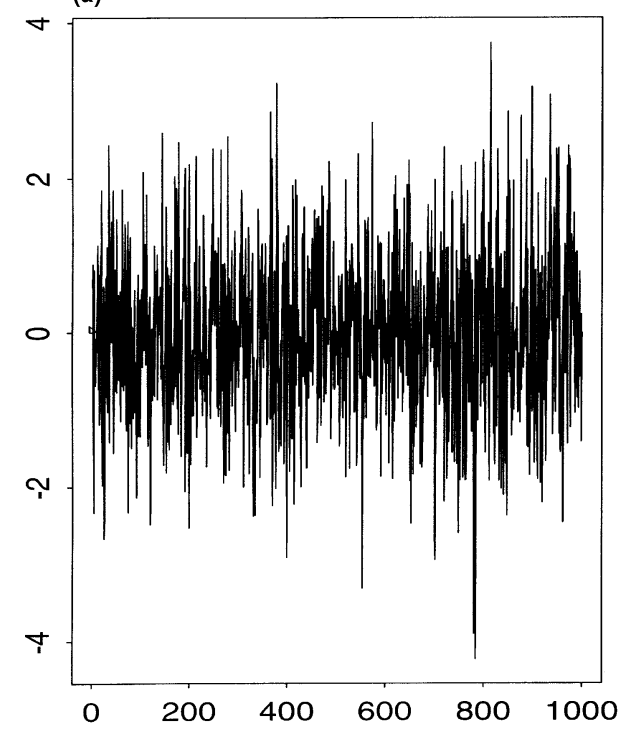

(b)

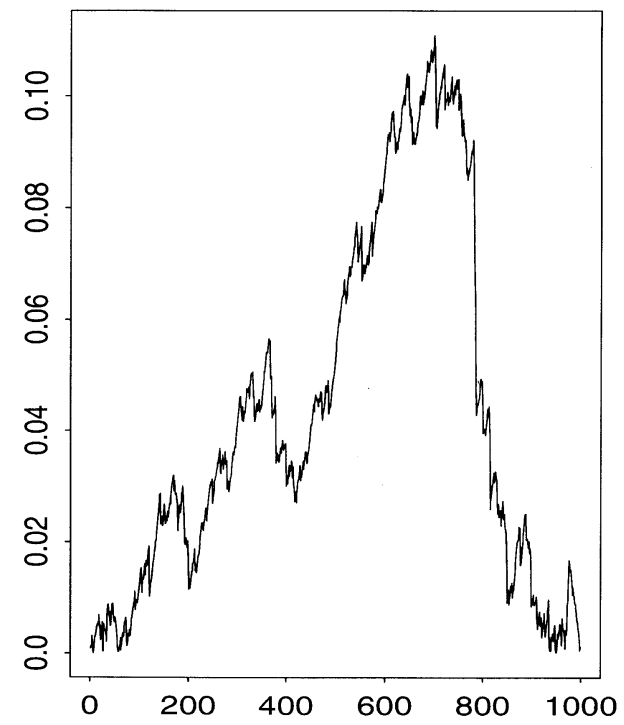

Figure 1. (a) A simulated realization of an $\mathrm{ARCH}(1)$ sequence with change-point at $k^{*}=700$; (b) the corresponding sequence $R_{k}$.

inequalities are available. Thus, in Section 4 we prove a rather general Hájek-Rényi type inequality which we believe is also of independent interest.

Section 5 contains the proof of the consistency of the estimator $\hat{k}$, which is fairly standard once the tools developed in Sections 3 and 4 are available. The rate of convergence of the estimator is established in Section 6. A small simulation study and a data example are discussed in Section 7.

\section{Stationarity of an $\operatorname{ARCH}(\infty)$ sequence}

In this section we recall the definition of an $\mathrm{ARCH}(\infty)$ sequence and give a simple sufficient condition for $\mathrm{ARCH}(\infty)$ equations (2.1) and (2.2) to have a weakly stationary solution. As far as we know, condition (2.3) below is new. Our method of proof is also different from the methods developed in Engle (1982) and Bollerslev (1986) and a large number of subsequent publications. It provides a closed-form solution to (2.1) and (2.2) and thus extends a similar result for ARCH(1) models; see, for example, Theorem 8.4.9 of Embrechts et al. (1997). Note also that we do not assume that the $\varepsilon_{k}$ in Definition 2.1 below are Gaussian but merely that they have finite fourth moment. Thus, condition (2.3) is also a sufficient condition for the existence of the fourth moments of the solution of equations (2.1) and (2.2). Necessary and sufficient conditions have recently been established by He and Teräsvirta (1997) for finiteorder GARCH models. 
We focus here on the squares of what is commonly referred to as an $\mathrm{ARCH}(\infty)$ sequence $r_{k}=\sigma_{k} \varepsilon_{k}$ with the conditional variances $\sigma_{k}^{2}$ defined by (2.2); in this paper the sequence of squares, $\left\{r_{k}^{2}\right\}$, is referred to as an $\mathrm{ARCH}$ process.

Definition 2.1. We say that a random sequence $\left\{r_{k}^{2}, k \in \mathbb{Z}\right\}$ satisfies $A R C H(\infty)$ equations if there exists a sequence of zero-mean i.i.d. random variables $\left\{\varepsilon_{k}, k \in \mathbb{Z}\right\}$ with $\mathrm{E} \varepsilon_{k}^{2}=1$ and $\mathrm{E} \varepsilon_{k}^{4}=\lambda<\infty$, such that

$$
\begin{gathered}
r_{k}^{2}=\sigma_{k}^{2} \varepsilon_{k}^{2}, \\
\sigma_{k}^{2}=a+\sum_{j=1}^{\infty} b(j) r_{k-j}^{2},
\end{gathered}
$$

where $a \geqslant 0, b(j) \geqslant 0, j=1,2, \ldots$

Theorem 2.1. Consider the quantities $B=\sum_{k=1}^{\infty} b(k)$ and $\lambda=\mathrm{E} \varepsilon_{k}^{4}$ as in Definition 2.1. If the condition

$$
\lambda B^{2}<1
$$

is satisfied, then ARCH( $\infty)$ equations (2.1) and (2.2) have a weakly stationary solution $\left\{r_{k}^{2}\right\}$ given by

$$
r_{k}^{2}=a Y_{k}
$$

where

$$
Y_{k}=\varepsilon_{k}^{2} \sum_{l=0}^{\infty} M(k, l)
$$

and

$$
M(k, l)=\sum_{j_{1} \ldots, j_{l}=1}^{\infty}\left(\prod_{m=1}^{l} \varepsilon_{k-j_{1}-\ldots-j_{m}}^{2} b\left(j_{m}\right)\right), l>0, \quad M(k, 0)=1 .
$$

We adopt the convention that $\prod_{l}^{l-i} a_{j}=1, \sum_{m}^{m-i} a_{j}=0$ for $i>0$, and denote $a \wedge b:=$ $\min \{a, b\}$.

Proof. We will show that $\left\{a Y_{k}\right\}$ is a second-order stationary sequence satisfying (2.1) and (2.2).

The assumption that $\mathrm{E} \varepsilon_{1}^{2}=1$ and the Cauchy inequality imply that $\lambda \geqslant 1$ and thus, by (2.3), $B<1$. Since all factors of the product $\prod_{m=1}^{l} \varepsilon_{k-j_{1}-\ldots-j_{m}}^{2}$ in (2.6) are independent, we obtain

$$
\mathrm{E} M(k, l)=\sum_{j_{1} \ldots, j_{l}=1}^{\infty} \prod_{m=1}^{l} b\left(j_{m}\right)=B^{l}
$$

and 


$$
\mathrm{E} Y_{k}=\sum_{l=0}^{\infty} \mathrm{E} M(k, l)=\sum_{l=0}^{\infty} B^{l}=\frac{1}{1-B}<\infty
$$

Therefore $M(k, l)$ and $Y_{k}$ are well defined: for any $k$ and $l, M(k, l)<\infty$ a.s. and $Y_{k}<\infty$ a.s. Using the recursive equality

$$
M(k, l)=\sum_{j=1}^{\infty} b(j) \varepsilon_{k-j}^{2} M(k-j, l-1),
$$

it is easy to verify that $\left\{a Y_{k}\right\}$ is a solution to (2.1) and (2.2).

Next we will show that the series $\sum_{l=1}^{\infty} M(k, l)$ converges in $L^{2}$. We use the Cauchy criterion and write

$$
\mathrm{E}\left|\sum_{n_{1}<l \leqslant n_{2}} M(k, l)\right|^{2}=\sum_{n_{1}<l, l^{\prime} \leqslant n_{2}} \operatorname{EM}(k, l) M\left(k, l^{\prime}\right),
$$

where

$\operatorname{EM}(k, l) M\left(k, l^{\prime}\right)$

$$
\begin{aligned}
& =\sum_{j_{1}, \ldots, j_{l}=1}^{\infty} \sum_{j_{1}^{\prime}, \ldots, j_{l^{\prime}}=1}^{\infty}\left[\left(\prod_{m=1}^{l} b\left(j_{m}\right)\right)\left(\prod_{m^{\prime}=1}^{l^{\prime}} b\left(j_{m^{\prime}}^{\prime}\right)\right) \mathrm{E}\left(\prod_{m=1}^{l} \varepsilon_{k-j_{1}-\ldots,-j_{m}}^{2} \prod_{m^{\prime}=1}^{l^{\prime}} \varepsilon_{k-j_{1}^{\prime}-\ldots-j_{m^{\prime}}}^{2}\right)\right] \\
& \leqslant \sum_{j_{1}, \ldots, j_{l}=1}^{\infty} \sum_{j_{1}^{\prime}, \ldots, j_{l^{\prime}}=1}^{\infty}\left[\left(\prod_{m=1}^{l} b\left(j_{m}\right)\right)\left(\prod_{m^{\prime}=1}^{l^{\prime}} b\left(j_{m^{\prime}}^{\prime}\right)\right)\left(\mathrm{E} \prod_{m=1}^{l} \varepsilon_{k-j_{1}-\ldots-j_{m}}^{4}\right)^{1 / 2}\left(\mathrm{E} \prod_{m^{\prime}=1}^{l^{\prime}} \varepsilon_{k-j_{1}^{\prime}-\ldots-j_{m^{\prime}}}^{4}\right)^{1 / 2}\right] \\
& =\lambda^{\left(l+l^{\prime}\right) / 2} B^{l+l^{\prime}} .
\end{aligned}
$$

This, combined with (2.3) and (2.7), yields

$$
\mathrm{E}\left|\sum_{n_{1}<l \leqslant n_{2}} M(k, l)\right|^{2} \rightarrow 0, \quad \text { as } n_{1}, n_{2} \rightarrow \infty .
$$

To verify that the sequence $r_{k}^{2}$ defined by (2.4) is weakly stationary, observe that

$$
\mathrm{E} Y_{k}=\frac{1}{1-B}=\sum_{l=0}^{\infty} \sum_{j_{1}, \ldots, j_{l}=1}^{\infty} \prod_{m=1}^{l} b\left(j_{m}\right)
$$

and hence 


$$
\begin{aligned}
& \operatorname{cov}\left(Y_{i}, Y_{i+k}\right)=\mathrm{E}\left(Y_{i} Y_{i+k}\right)-\mathrm{E} Y_{i} \mathrm{E} Y_{i+k} \\
& =\sum_{l, l^{\prime}=0}^{\infty} \sum_{j_{1}^{\prime}, \ldots, j_{l^{\prime}}=1}^{\infty}\left(\prod_{m^{\prime}=1}^{l^{\prime}} b\left(j_{m^{\prime}}^{\prime}\right)\right) \sum_{j_{1}, \ldots, j_{l}=1}^{\infty}\left(\prod_{m=1}^{l} b\left(j_{m}\right)\right) \mathrm{E}\left(\prod_{m^{\prime}=0}^{l^{\prime}} \varepsilon_{i-j_{1}^{\prime}-\ldots-j_{m^{\prime}}}^{2} \prod_{m=0}^{l} \varepsilon_{i+k-j_{1}-\ldots-j_{m}}^{2}-1\right) \\
& =\operatorname{cov}\left(Y_{0}, Y_{k}\right) .
\end{aligned}
$$

\section{The cross-covariance function of $\operatorname{ARCH}(\infty)$ sequences}

In order to find the correlation function (of the squares) of an $\operatorname{ARCH}(p)$ or $\operatorname{GARCH}(p, q)$ sequence with finite $p$ and $q$, equalities analogous to Yule-Walker equations have been used in the existing literature. For example, for the $\mathrm{ARCH}(1)$ sequence

$$
r_{k}^{2}=\left(a+b r_{k-1}^{2}\right) \varepsilon_{k}^{2}
$$

multiplying both sides of (3.1) by $r_{0}^{2}$, taking expectations and using the relation $\mathrm{E} r_{k}^{2}=$ $a /(1-b)$, we obtain $\operatorname{cov}\left(r_{0}^{2}, r_{k}^{2}\right)=b^{k} \operatorname{var} r_{0}^{2}$. However, computing the variance $\operatorname{var} r_{0}^{2}$ is not easy and requires a representation of $r_{0}^{2}$ in terms of the $\varepsilon_{k}^{2}$.

Similarly, in the $\operatorname{GARCH}(p, q)$ case, one can derive Yule-Walker type equalities (see Bollerslev, 1986) which can be solved when the first $q+1$ covariances are known.

In this section we focus on the cross-covariance function of two $\mathrm{ARCH}(\infty)$ sequences $\left\{r_{1, k}^{2}, k \in \mathbb{Z}\right\}$ and $\left\{r_{2, k}^{2}, k \in \mathbb{Z}\right\}$ driven by the same sequence $\left\{\varepsilon_{k}\right\}$ of i.i.d. random variables with $\mathrm{E} \varepsilon_{1}=0, \mathrm{E} \varepsilon_{1}^{2}=1$ and $\mathrm{E} \varepsilon_{1}^{4}=\lambda<\infty$, that is,

$$
r_{i, k}^{2}=\sigma_{i, k}^{2} \varepsilon_{k}^{2}, \quad \sigma_{i, k}^{2}=a_{i}+\sum_{j=1}^{\infty} b_{i}(j) r_{i, k-j}^{2}, \quad i=1,2,
$$

where $a_{i} \geqslant 0$, and $\left\{b_{i}(j)\right\}, i=1,2$, are two sequences of non-negative numbers.

Our results are valid for 'short memory' $\operatorname{ARCH}(\infty)$ models, that is to say, we assume that the $b_{i}(j)$ decay exponentially fast. More specifically, we suppose in the remainder of the paper that the following assumption holds:

Assumption 3.1. The coefficients $b(j)$ in Definition 2.1 satisfy

$$
b(j) \leqslant \beta \alpha^{j}
$$

for some $0 \leqslant \alpha<1$ and $\beta \geqslant 0$ such that

$$
\lambda\left(\frac{\beta \alpha}{1-\alpha}\right)^{2}<1
$$

Clearly, condition (3.4) implies (2.3).

In the proof of Theorem 3.1 and thereafter we will extensively use the quantities 


$$
B_{i}(k):=b_{i}(k)+b_{i}(k+1)+\ldots \quad \text { and } \quad B_{i}:=B_{i}(1), \quad i=1,2 .
$$

We now formulate the main theorem of this section.

Theorem 3.1. If the sequences $\left\{r_{1, k}^{2}\right\}$ and $\left\{r_{2, k}^{2}\right\}$ satisfy Assumption 3.1, then

$$
0 \leqslant \operatorname{cov}\left(r_{1, k}^{2}, r_{2, k^{\prime}}^{2}\right) \leqslant a_{1} a_{2} C_{1} \alpha^{\left|k^{\prime}-k\right|}(1+\beta)^{\left|k^{\prime}-k\right|},
$$

where

$$
C_{1}=\frac{\beta(\lambda-1)}{(1+\beta)(1-\alpha)\left(1-B_{1}\right)\left(1-B_{2}\right)\left(1-B_{1} B_{2} \lambda\right)} .
$$

Note that, since $\lambda \geqslant 1$, condition (3.4) implies $\alpha(1+\beta)<1$.

Proof. Set $r_{1, k}^{2}=a_{1} Y_{k}^{(1)}, \quad r_{2, k}^{2}=a_{2} Y_{k}^{(2)}$, where the $Y_{k}^{(i)}$ are defined by (2.5) with corresponding parameters $b_{i}(1), b_{i}(2), \ldots$ Note that

$$
\begin{aligned}
& \operatorname{cov}\left(Y_{k}^{(1)}, Y_{k^{\prime}}^{(2)}\right) \\
& =\sum_{l, l^{\prime}=0}^{\infty} \sum_{j^{\prime}, \ldots, j_{l^{\prime}}=1}^{\infty}\left(\prod_{m^{\prime}=1}^{l^{\prime}} b_{2}\left(j_{m^{\prime}}^{\prime}\right)\right) \sum_{j_{1}, \ldots, j_{l}=1}^{\infty}\left(\prod_{m=1}^{l} b_{1}\left(j_{m}\right)\right) \mathrm{E}\left(\prod_{m^{\prime}=0}^{l^{\prime}} \varepsilon_{k^{\prime}-j_{1}^{\prime}-\ldots-j_{m^{\prime}}}^{2} \prod_{m=0}^{l} \varepsilon_{k-j_{1}-\ldots-j_{m}}^{2}-1\right) .
\end{aligned}
$$

Assume first that $k>k^{\prime}$. Then the products $\prod_{m^{\prime}=0}^{l^{\prime}} \varepsilon_{k^{\prime}-j_{1}^{\prime}-\ldots-j_{m^{\prime}}}^{2}$ and $\prod_{m=0}^{l} \varepsilon_{k-j_{1}-\ldots-j_{m}}^{2}$ in (3.7) are independent if $l=0$ or $j_{1}+\ldots+j_{l}<k-k^{\prime}$. Thus we can write

$$
\begin{aligned}
\operatorname{cov}\left(Y_{k}^{(1)}, Y_{k^{\prime}}^{(2)}\right)= & \sum_{\substack{l=1 \\
l^{\prime}=0}}^{\infty} \sum_{j_{1}^{\prime}, \ldots, j_{l^{\prime}}=1}^{\infty}\left(\prod_{m^{\prime}=1}^{l^{\prime}} b_{2}\left(j_{m^{\prime}}^{\prime}\right)\right) \sum_{\substack{j_{1}, \ldots, j_{l}=1 \\
j_{1}+\ldots+j_{l} \geqslant k-k^{\prime}}}^{\infty}\left(\prod_{m=1}^{l} b_{1}\left(j_{m}\right)\right) \\
& \times \mathrm{E}\left(\prod_{m^{\prime}=0}^{l^{\prime}} \varepsilon_{k^{\prime}-j_{1}^{\prime}-\ldots-j_{m^{\prime}}}^{2} \prod_{m=0}^{l} \varepsilon_{k-j_{1}-\ldots-j_{m}}^{2}-1\right) .
\end{aligned}
$$

We now split the last sum in (3.8) into a sum over $j_{1}+\ldots+j_{l-1} \geqslant k-k^{\prime}$ and a sum over $j_{1}+\ldots+j_{l-1}<k-k^{\prime}$. This procedure is carried on until the second sum is split into $l$ sums $S_{1}, \ldots, S_{l}$ (see (3.9) and (3.10) below), such that $S_{i}$ can be appropriately bounded above (see (3.16)). The latter bound is then used in (3.17) and (3.18), which lead to (3.5). Write, then, 


$$
\begin{aligned}
& \sum_{\substack{j_{1}, \ldots, j_{l}=1 \\
j_{1}+\ldots+j_{l} \geqslant k-k^{\prime}}}^{\infty}\left(\prod_{m=1}^{l} b_{1}\left(j_{m}\right)\right) \mathrm{E}\left(\prod_{m^{\prime}=0}^{l^{\prime}} \varepsilon_{k^{\prime}-j_{1}^{\prime}-\ldots-j_{m^{\prime}}}^{2} \prod_{m=0}^{l} \varepsilon_{k-j_{1}-\ldots-j_{m}}^{2}-1\right) \\
= & \sum_{\substack{j_{1}, \ldots, j_{l}=1 \\
j_{1}+\ldots+j_{l-1} \geqslant k-k^{\prime}}}^{\infty}\left(\prod_{m=1}^{l} b_{1}\left(j_{m}\right)\right) \mathrm{E}\left(\prod_{m^{\prime}=0}^{l^{\prime}} \varepsilon_{k^{\prime}-j_{1}^{\prime}-\ldots-j_{m^{\prime}}}^{2} \prod_{m=0}^{l} \varepsilon_{k-j_{1}-\ldots-j_{m}}^{2}-1\right) \\
+ & \sum_{\substack{j_{1}, \ldots, j_{l-1}=1 \\
j_{1}+\ldots+j_{l-1}<k-k^{\prime}}}^{\infty}\left(\prod_{m=1}^{l-1} b_{1}\left(j_{m}\right)\right)_{j_{l}=k-k^{\prime}-j_{1}-\ldots-j_{l-1}} b_{1}\left(j_{l}\right) \mathrm{E}\left(\prod_{m^{\prime}=0}^{l^{\prime}} \varepsilon_{k^{\prime}-j_{1}^{\prime}-\ldots-j_{m^{\prime}}^{\prime}}^{2} \prod_{m=0}^{l} \varepsilon_{k-j_{1}-\ldots-j_{m}}^{2}-1\right) \\
= & : T+S_{1} .
\end{aligned}
$$

For the term $T$, consider analogously the cases $j_{1}+\ldots+j_{l-2} \geqslant k-k^{\prime}$ and $j_{1}+\ldots+$ $j_{l-2}<k-k^{\prime}$ :

$$
\begin{aligned}
T= & \sum_{\substack{j_{1}, \ldots, j_{l}=1 \\
j_{1}+\ldots+j_{l-2} \geqslant k-k^{\prime}}}^{\infty}\left(\prod_{m=1}^{l} b_{1}\left(j_{m}\right)\right) \mathrm{E}\left(\prod_{m^{\prime}=0}^{l^{\prime}} \varepsilon_{k^{\prime}-j^{\prime}-\ldots-j_{m^{\prime}}}^{2} \prod_{m=0}^{l} \varepsilon_{k-j_{1}-\ldots-j_{m}}^{2}-1\right) \\
& +\sum_{\substack{j_{1}, \ldots, j_{l-2}=1 \\
j_{1}+\ldots+j_{l-2}<k-k^{\prime}}}^{\infty}\left(\prod_{m=1}^{l-2} b_{1}\left(j_{m}\right)\right) \prod_{j_{l-1}=k-k} \prod_{k^{\prime}-j_{1}-\ldots-j_{l-2}}^{\infty} b_{1}\left(j_{l-1}\right) \sum_{j_{l}=1}^{\infty} b_{1}\left(j_{l}\right) \\
& \times \mathrm{E}\left(\prod_{m^{\prime}=0}^{l^{\prime}} \varepsilon_{k^{\prime}-j_{1}^{\prime}-\ldots-j_{m^{\prime}}}^{2} \prod_{m=0}^{l} \varepsilon_{k-j_{1}-\ldots-j_{m}}^{2}-1\right) .
\end{aligned}
$$

Continuing the above procedure, we obtain

$$
T=S_{2}+\ldots+S_{l}
$$

where

$$
\begin{aligned}
S_{i}= & \sum_{\substack{j_{1}, \ldots, j_{l-i}=1 \\
j_{1}+\ldots+j_{l-i}<k-k^{\prime}}}^{\infty}\left(\prod_{m=1}^{l-i} b_{1}\left(j_{m}\right)\right) \sum_{j_{l-i+1}=k-k^{\prime}-j_{1}-\ldots-j_{l-i}}^{\infty} b_{1}\left(j_{l-i+1}\right) \sum_{j_{l-i+2}=1}^{\infty} b_{1}\left(j_{l-i+2}\right) \ldots \sum_{j_{l}=1}^{\infty} b_{1}\left(j_{l}\right) \\
& \times \mathrm{E}\left(\prod_{m^{\prime}=0}^{l^{\prime}} \varepsilon_{k^{\prime}-j^{\prime}-\ldots-j_{m^{\prime}}}^{2} \prod_{m=0}^{l} \varepsilon_{k-j_{1}-\ldots-j_{m}}^{2}-1\right) .
\end{aligned}
$$

For the term $S_{l}$ we can write 


$$
\begin{aligned}
S_{l} & \leqslant \sum_{j_{1}=k-k^{\prime}}^{\infty} b_{1}\left(j_{1}\right) \sum_{j_{2}=1}^{\infty} b_{1}\left(j_{2}\right) \ldots \sum_{j_{l}=1}^{\infty} b_{1}\left(j_{l}\right)\left(\lambda^{\left(l^{\prime}+1\right) \wedge l}-1\right) \\
& =B_{1}\left(k-k^{\prime}\right) B_{1}^{l-1}\left(\lambda^{\left(l^{\prime}+1\right) \wedge l}-1\right) .
\end{aligned}
$$

For the terms $S_{i}(i=1, \ldots, l-1)$ we obtain

$$
\begin{aligned}
& S_{i}=\sum_{\substack{j_{1}, \ldots, j_{l-i}=1 \\
j_{1}+\ldots+j_{l-i}<k-k^{\prime}}}^{\infty}\left(\prod_{m=1}^{l-i} b_{1}\left(j_{m}\right)\right)_{j_{l-i+1}=k-k^{\prime}-j_{1}-\ldots-j_{l-i}}^{\infty} b_{1}\left(j_{l-i+1}\right) \sum_{j_{l-i+2}=1}^{\infty} b_{1}\left(j_{l-i+2}\right) \ldots \sum_{j_{l}=1}^{\infty} b_{1}\left(j_{l}\right) \\
& \times \mathrm{E}\left(\prod_{m^{\prime}=0}^{l^{\prime}} \varepsilon_{k^{\prime}-j_{1}^{\prime}-\ldots-j_{m^{\prime}}^{\prime}}^{2} \prod_{m=l-i+1}^{l} \varepsilon_{k-j_{1}-\ldots-j_{m}}^{2}-1\right) . \\
& \leqslant \sum_{\substack{j_{1}, \ldots, j_{l-i}=1 \\
j_{1}+\ldots+j_{l-i}<k-k^{\prime}}}^{\infty}\left(\prod_{m=1}^{l-i} b_{1}\left(j_{m}\right)\right) \sum_{j_{l-i+1}=k-k^{\prime}-j_{1}-\ldots-j_{l-i}}^{\infty} b_{1}\left(j_{l-i+1}\right) \ldots \sum_{j_{l}=1}^{\infty} n_{1}\left(j_{l}\right)\left(\lambda^{\left(l^{\prime}+1\right) \wedge i}-1\right) \\
& =\left(\lambda^{\left(l^{\prime}+1\right) \wedge i}-1\right) B^{i-1} \sum_{\substack{j_{1}, \ldots, j_{l-i}=1 \\
j_{1}+\ldots+j_{l-i}<k-k^{\prime}}}^{\infty}\left(\prod_{m=1}^{l-i} b_{1}\left(j_{m}\right)\right)_{j_{l-i+1}=k-k^{\prime}-j_{1}-\ldots-j_{l-i}}^{\infty} b_{1}\left(j_{l-i+1}\right) .
\end{aligned}
$$

By the assumption $b_{i}(j) \leqslant \beta \alpha^{j}$,

$$
\sum_{j_{l-i+1}=k-k^{\prime}-j_{1}-\ldots-j_{l-i}}^{\infty}\left(\prod_{m=1}^{l-i} b_{1}\left(j_{m}\right)\right) b_{1}\left(j_{l-i+1}\right) \leqslant \beta^{l-i+1} \frac{\alpha^{k-k^{\prime}}}{1-\alpha} .
$$

Thus, for $i=1, \ldots, l-1$,

$$
S_{i} \leqslant\left(\lambda^{\left(l^{\prime}+1\right) \wedge i}-1\right) B_{1}^{i-1} \beta^{l-i} A_{k-k^{\prime}} \sum_{\substack{j_{1}, \ldots, j_{l-i}=1 \\ j_{1}+\ldots+j_{l-i}<k-k^{\prime}}}^{\infty} 1,
$$

where

$$
A_{k}:=\frac{\beta \alpha^{k}}{1-\alpha} \text {. }
$$

Since

$$
\sum_{\substack{j_{1}, \ldots, j_{l}=1 \\
j_{1}+\ldots+j_{l}=m}}^{\infty} 1=\left(\begin{array}{c}
m-1 \\
l-1
\end{array}\right)
$$

we have 


$$
\sum_{\substack{j_{1}, \ldots, j_{l-i}=1 \\
j_{1}+\ldots+j_{l-i}<k-k^{\prime}}}^{\infty} 1=\sum_{j=0}^{k-k^{\prime}-1+i-1}\left(\begin{array}{c}
j+l-i-1 \\
j
\end{array}\right) 1_{\left\{l-i<k-k^{\prime}\right\}} .
$$

By (3.14) and (3.15) it follows that, for $i=1, \ldots, l-1$,

$$
S_{i} \leqslant\left(\lambda^{\left(l^{\prime}+1\right) \wedge i}-1\right) B_{1}^{i-1} \beta^{l-i} A_{k-k^{\prime}} \sum_{j=0}^{k-k^{\prime}-l+i-1}\left(\begin{array}{c}
j+l-i-1 \\
j
\end{array}\right) 1_{\left\{l-i<k-k^{\prime}\right\}} .
$$

Now substitute these estimates to (3.10), (3.9) and (3.8) and write

$$
\begin{aligned}
& \left.\operatorname{cov} Y_{k}^{(1)}, Y_{k^{\prime}}^{(2)}\right) \leqslant \sum_{\substack{l=1 \\
l^{\prime}=0}}^{\infty} \sum_{j^{\prime}, \ldots, j_{l^{\prime}}=1}^{\infty}\left(\prod_{m^{\prime}=1}^{l^{\prime}} b_{2}\left(j_{m^{\prime}}^{\prime}\right)\right) S_{l}+\sum_{\substack{l=1 \\
l^{\prime}=0}}^{\infty} \sum_{\substack{j^{\prime} \\
n_{1}, \ldots, j_{l^{\prime}}=1}}^{\infty}\left(\prod_{m^{\prime}=1}^{l^{\prime}} b_{2}\left(j_{m^{\prime}}^{\prime}\right)\right) \sum_{i=1}^{l-1} S_{i} \\
& =: s_{1}+s_{2} \text {. }
\end{aligned}
$$

Consider first the term $s_{1}$ :

$$
\begin{aligned}
s_{1} & =\sum_{l^{\prime}=0}^{\infty} B_{2}^{l^{\prime}} \sum_{l=1}^{\infty} S_{l} \leqslant B_{1}\left(k-k^{\prime}\right)\left[\sum_{l^{\prime}=0}^{\infty} B_{2}^{l^{\prime}} \sum_{l=1}^{\infty} B_{1}^{l-1} \lambda^{\left(l^{\prime}+1\right) \wedge l}-\sum_{l^{\prime}=0}^{\infty} B_{2}^{l^{\prime}} \sum_{l=1}^{\infty} B_{1}^{l-1}\right] \\
& =B_{1}\left(k-k^{\prime}\right)\left[\sum_{l^{\prime}=0}^{\infty} B_{2}^{l^{\prime}} \sum_{l=1}^{l^{\prime}+1} B_{1}^{l-1} \lambda^{l}+\sum_{l^{\prime}=0}^{\infty} B_{2}^{l^{\prime}} \sum_{l=l^{\prime}+2}^{\infty} B_{1}^{l-1} \lambda^{l^{\prime}+1}-\left(1-B_{1}\right)^{-1}\left(1-B_{2}\right)^{-1}\right] \\
& =B_{1}\left(k-k^{\prime}\right)\left[\sum_{l=1}^{\infty} B_{1}^{l-1} \lambda^{l} \sum_{l^{\prime}=l-1}^{\infty} B_{2}^{l^{\prime}}+\sum_{l^{\prime}=0}^{\infty} B_{2}^{l^{\prime}} \lambda^{l^{\prime}+1} \sum_{l=l^{\prime}+2}^{\infty} B_{1}^{l-1}-\left(1-B_{1}\right)^{-1}\left(1-B_{2}\right)^{-1}\right] \\
& =B_{1}\left(k-k^{\prime}\right)\left[\frac{\lambda}{\left(1-B_{2}\right)\left(1-B_{1} B_{2} \lambda\right)}+\frac{B_{1} \lambda}{\left(1-B_{1}\right)\left(1-B_{1} B_{2} \lambda\right)}-\frac{1}{\left(1-B_{1}\right)\left(1-B_{2}\right)}\right] \\
& =B_{1}\left(k-k^{\prime}\right) \frac{\lambda-1}{\left(1-B_{1}\right)\left(1-B_{2}\right)\left(1-B_{1} B_{2} \lambda\right)} .
\end{aligned}
$$

For the term $s_{2}$ we have, by (3.16),

$$
\begin{aligned}
s_{2} \leqslant & A_{k-k^{\prime}} \sum_{\substack{l=1 \\
l^{\prime}=0}}^{\infty} \sum_{j_{1}^{\prime}, \ldots, j_{l^{\prime}}=1}^{\infty}\left(\prod_{m^{\prime}=1}^{l^{\prime}} b_{2}\left(j_{m^{\prime}}^{\prime}\right)\right) \\
& \times \sum_{i=1}^{l-1} \beta^{l-i}\left(\lambda^{\left(l^{\prime}+1\right) \wedge i}-1\right) B_{1}^{i-1} \sum_{j=0}^{k-k^{\prime}-l+i-1}\left(\begin{array}{c}
j-i-1 \\
j
\end{array}\right) 1_{\left\{l-i<k-k^{\prime}\right\}} \\
= & A_{k-k^{\prime}} \sum_{l^{\prime}=0}^{\infty} B_{2}^{l^{\prime}} \sum_{i=1}^{\infty}\left(\lambda^{\left(l^{\prime}+1\right) \wedge i}-1\right) B_{1}^{i-} G_{\beta}\left(k-k^{\prime}\right)
\end{aligned}
$$


where

$$
\begin{aligned}
G_{\beta}(k) & :=\sum_{l=i+1}^{i+k-1} \beta^{l-i} \sum_{j=0}^{k-l+i-1}\left(\begin{array}{c}
j+l-i-1 \\
j
\end{array}\right) \\
& =\sum_{m=1}^{k-1} \beta^{m} \sum_{j=0}^{k-m-1}\left(\begin{array}{c}
j+m-1 \\
j
\end{array}\right)=\sum_{m=1}^{k-1} \beta^{m}\left(\begin{array}{c}
k-1 \\
m
\end{array}\right) .
\end{aligned}
$$

It is easy to see that $G_{\beta}(1)=0, G_{\beta}(2)=\beta, \ldots, G_{\beta}(k)=G_{\beta}(k-1)+\beta(\beta=1)^{k-2}, k \geqslant 2$, and hence

$$
G_{\beta}(k)=(\beta+1)^{k-1}-1 .
$$

Using (3.18), we now establish an upper bound for $s_{2}$ as follows:

$$
\begin{aligned}
s_{2} & \leqslant A_{k-k^{\prime}} G_{\beta}\left(k-k^{\prime}\right)\left[\sum_{l^{\prime}=0}^{\infty} B_{2}^{l^{\prime}} \sum_{i=1}^{l^{\prime}} \lambda^{i} B_{1}^{i-1}+\sum_{l^{\prime}=0}^{\infty} B_{2}^{l^{\prime}} \sum_{i=l^{\prime}+1}^{\infty} \lambda^{l^{\prime}+1} B_{1}^{i-1}-\sum_{l^{\prime}=0}^{\infty} B_{2}^{l^{\prime}} \sum_{i-1}^{\infty} B_{1}^{i-1}\right] \\
& =A_{k-k^{\prime}} G_{\beta}\left(k-k^{\prime}\right)\left[\sum_{i=1}^{\infty} \lambda^{i} B_{1}^{i-1} \sum_{l^{\prime}=i}^{\infty} B_{2}^{l^{\prime}}+\sum_{l^{\prime}=0}^{\infty} B_{2}^{l^{\prime}} \lambda^{l^{\prime}+1} \sum_{i=l^{\prime}+1}^{\infty}-\frac{1}{\left(1-B_{1}\right)\left(1-B_{2}\right)}\right] \\
& =A_{k-k^{\prime}} G_{\beta}\left(k-k^{\prime}\right)\left[\sum_{i=1}^{\infty} \lambda^{i} B_{1}^{i-1}\left(1-B_{2}\right)^{-1} B_{2}^{i}+\sum_{l^{\prime}=0}^{\infty} B_{2}^{l^{\prime}} \lambda^{l^{\prime}+1}\left(1-B_{1}\right)^{-1} B_{1}^{l^{\prime}}-\frac{1}{\left(1-B_{1}\right)\left(1-B_{2}\right)}\right] \\
& =A_{k-k^{\prime}} G_{\beta}\left(k-k^{\prime}\right)\left[\frac{\lambda B_{2}}{\left(1-B_{2}\right)\left(1-B_{1} B_{2} \lambda\right)}+\frac{\lambda}{\left(1-B_{1}\right)\left(1-B_{1} B_{2} \lambda\right)}-\frac{1}{\left(1-B_{1}\right)\left(1-B_{2}\right)}\right] \\
& =\alpha^{k-k^{\prime}} G_{\beta}\left(k-k^{\prime}\right) \frac{\beta(\lambda-1)}{(1-\alpha)\left(1-B_{1}\right)\left(1-B_{2}\right)\left(1-B_{1} B_{2} \lambda\right)} .
\end{aligned}
$$

Applying now (3.17) and (3.19), we obtain

$$
\begin{aligned}
\operatorname{cov}\left(Y_{k}^{(1)}, Y_{k^{\prime}}^{(2)}\right) \leqslant & B_{1}\left(k-k^{\prime}\right) \frac{\lambda-1}{\left(1-B_{1}\right)\left(1-B_{2}\right)\left(1-B_{1} B_{2} \lambda\right)} \\
& +\alpha^{k-k^{\prime}} G_{\beta}\left(k-k^{\prime}\right) \frac{\beta(\lambda-1)}{(1-\alpha)\left(1-B_{1}\right)\left(1-B_{2}\right)\left(1-B_{1} B_{2} \lambda\right)} \\
= & \frac{\lambda-1}{\left(1-B_{1}\right)\left(1-B_{2}\right)\left(1-B_{1} B_{2} \lambda\right)}\left(B_{1}\left(k-k^{\prime}\right)+\frac{\beta}{1-\alpha} \alpha^{k-k^{\prime}} G_{\beta}\left(k-k^{\prime}\right)\right) .
\end{aligned}
$$

The inequality $B_{1}\left(k-k^{\prime}\right) \leqslant \beta \alpha^{k-k^{\prime}}(1-\alpha)^{-1}$ yields (3.5).

So far we have been assuming that $k>k^{\prime}$ in order to ensure the independence of certain products in (3.7). The case $k^{\prime}>k$ is analogous. If $k=k^{\prime}$, observe first that setting $a_{i}=1$ yields $Y_{k}^{(i)}=r_{i, k}^{2}$. Then the claim follows from the general identity 


$$
\operatorname{cov}\left(r_{1, k}^{2}, r_{2, k}^{2}\right) \sum_{j=1}^{\infty} b_{1}(j) \operatorname{cov}\left(r_{1, k-j}^{2}, r_{2, k}^{2}\right),
$$

which can be obtained by multiplying both sides of

$$
r_{1, k}^{2}=a_{1}+\sum_{j=1}^{\infty} b_{1}(j) r_{1, k-j}^{2}
$$

by $r_{2, k}^{2}$, taking expectations, and using the identity $\mathrm{E} r_{i, k}^{2}=a_{i} /\left(1-B_{i}\right)$. Then use the relation

$$
\operatorname{cov}\left(r_{1, k-j}^{2}, r_{2, k}^{2}\right)=\operatorname{cov}\left(r_{1, j}^{2}, r_{2,0}^{2}\right) \leqslant C_{1}(\alpha(1+\beta))^{j}
$$

and (3.5) with $k-k^{\prime}=j>0$.

Remark 3.1. Careful examination of the proof of Theorem 3.1 reveals that Assumption 3.1 can be replaced by the condition

$$
b_{i}\left(j^{\prime}\right) b_{i}(j) \leqslant \beta_{i} b_{i}\left(j^{\prime}+j\right), \quad i=1,2 .
$$

Using (3.22) in (3.13) instead of Assumption 3.1, we obtain the following inequalities

$$
\operatorname{cov}\left(Y_{k}^{(1)}, Y_{k^{\prime}}^{(2)}\right) \leqslant \begin{cases}\frac{\lambda-1}{\left(1-B_{1}\right)\left(1-B_{2}\right)\left(1-B_{1} B_{2} \lambda\right)} B_{1}\left(k-k^{\prime}\right)\left(1+\beta_{1}\right)^{k-k^{\prime}-1}, & \text { if } k \geqslant k^{\prime}, \\ \frac{\lambda-1}{\left(1-B_{1}\right)\left(1-B_{2}\right)\left(1-B_{1} B_{2} \lambda\right)} B_{2}\left(k^{\prime}-k\right)\left(1+\beta_{2}\right)^{k^{\prime}-k-1}, & \text { if } k<k^{\prime},\end{cases}
$$

where, roughly speaking, the factors $\alpha^{\left|k-k^{\prime}\right|}$ in (3.5) are replaced by the tails $B_{i}\left(\left|k-k^{\prime}\right|\right)$.

Remark 3.2. Replacing $Y_{k^{\prime}}^{(2)}$ by $Y_{k}^{(1)}$ in (3.5), a bound on the covariance function of the sequence $\left\{Y_{k}\right\}$ defined by (2.5) is obtained. The rate $\alpha^{k}(1+\beta)^{k}$ is optimal under Assumption 3.1. This can be verified by setting $b_{j}=\beta \alpha^{j}$, which corresponds to considering a $\operatorname{GARCH}(1,1)$ model as in Bollerslev (1988), who verified an analogous result for the correlation function of $\operatorname{GARCH}(1,1)$ models with normal errors. Obtaining a formula for the covariance function of GARCH models is much more complicated; see He and Teräsvirta (1997).

Remark 3.3. Using the technique developed in the proof of Theorem 3.1, it is very easy to verify that for the $\mathrm{ARCH}(1)$ sequence $r_{k}^{2}=\left(a+b r_{k-1}^{2}\right) \varepsilon_{k}^{2}$ or, equivalently, $r_{k}^{2}=a Y_{k}$, where $Y_{k}=\sum_{j=0}^{\infty} b^{j} \prod_{m=0}^{j} \varepsilon_{k-m}^{2}$,

$$
\operatorname{cov}\left(Y_{0}, Y_{k}\right)=K b^{k}
$$

where

$$
K=\frac{\lambda-1}{\left(1-b^{2} \lambda\right)(1-b)^{2}} .
$$

This follows from the equality 


$$
\begin{aligned}
\operatorname{cov}\left(Y_{0}, Y_{k}\right) & =\mathrm{E} \sum_{j, j^{\prime}=0}^{\infty} b^{j+j^{\prime}}\left(\prod_{m=0}^{j} \varepsilon_{-m}^{2} \prod_{m^{\prime}=0}^{j^{\prime}} \varepsilon_{k-m^{\prime}}^{2}-1\right) \\
& =\sum_{j=0}^{\infty} \sum_{i=0}^{j} b^{j} b^{k+i}\left(\lambda^{i+1}-1\right)+\sum_{j=0}^{\infty} \sum_{i=j+1}^{\infty} b^{j} b^{k+i}\left(\lambda^{j+1}-1\right) .
\end{aligned}
$$

We have assumed so far that the sequences $\left\{r_{1, k}^{2}\right\}$ and $\left\{r_{2, k}^{2}\right\}$ start infinitely far in the past. After some modifications our methods can, however, also be applied to sequences which start at some initial time point. To illustrate the idea, we focus here only on $\mathrm{ARCH}(1)$ models. Assume, then, that sequences $\left\{r_{1, k}^{2}, k=0,1, \ldots\right\}$ and $\left\{r_{2, k}^{2}, k=0\right.$, $1, \ldots\}$ are defined as follows:

$$
r_{i, k}^{2}=\sigma_{i, k}^{2} \varepsilon_{k}^{2}, \quad \sigma_{i, k}^{2}=a_{i}+b_{i} r_{i, k-1}^{2}, \quad k \geqslant 1,
$$

where $a_{i} \geqslant 0,0 \leqslant b_{i}<1$ and $b_{i}^{2} \lambda<1, i=1,2$, which is precisely condition (2.3) of Theorem 2.1. The initial values $r_{1,0}$ and $r_{2,0}$, such that $\mathrm{E} r_{i, 0}^{4}<\infty$, are assumed to be independent of $\left\{\varepsilon_{t}\right\}$, but not necessary mutually independent. The following result gives a closed-form formula for the cross-covariance function of the sequences $\left\{r_{1, k}^{2}\right\}$ and $\left\{r_{2, k}^{2}\right\}$ starting at $k=0$. The covariance function of each sequence $\left\{r_{i, k}^{2}\right\}$ can be obtained by setting $V=0$ in (3.26) and replacing $a_{1} a_{2}$ by $a_{i}^{2}$.

Theorem 3.2. For the sequences $\left\{r_{1, k}^{2}\right\}$ and $\left\{r_{2, k}^{2}\right\}$ given in (3.25) the cross-covariance function is

$$
\operatorname{cov}\left(r_{1, k}^{2}, r_{2, k^{\prime}}^{2}\right)= \begin{cases}U b_{2}^{k^{\prime}-k}+V\left(b_{1} \lambda\right)^{k} b_{2}^{k^{\prime}}, & \text { if } k \leqslant k^{\prime} \\ U b_{1}^{k-k^{\prime}}+V\left(b_{2} \lambda\right)^{k^{\prime}} b_{1}^{k}, & \text { if } k>k^{\prime}\end{cases}
$$

where

$$
\begin{aligned}
& U=\frac{a_{1} a_{2}(\lambda-1)}{\left(1-b_{1}\right)\left(1-b_{2}\right)\left(1-b_{1} b_{2} \lambda\right)}, \\
& V=-\frac{a_{1} a_{2}\left(1-b_{1} b_{2}\right) \lambda}{\left(1-b_{1}\right)\left(1-b_{2}\right)\left(1-b_{1} b_{2} \lambda\right)}+\mathrm{E}\left(r_{1,0}^{2} r_{2,0}^{2}\right) .
\end{aligned}
$$

The proof is given in the Appendix.

\section{A Hájek-Rényi type inequality}

In this section we prove a Hájek-Rényi type inequality, that is, an inequality for the maximum of weighted sums $c_{k}\left|X_{1}+\ldots+X_{k}\right|$, where $X_{1}, \ldots, X_{n}$ are any finite-variance random variables. The result is stated in Theorem 4.1 below. In the proof we will need an elementary inequality, which we now state as a lemma, as we could not find any reference to it. 
Lemma 4.1. For any random variables $Y_{1}, \ldots, Y_{n}$ and events $A=\left\{\max _{1 \leqslant k \leqslant n} Y_{k}>\varepsilon\right\}, D_{k}=$ $\left\{Y_{1} \leqslant \varepsilon, \ldots, Y_{k} \leqslant \varepsilon\right\}$

$$
\varepsilon \mathbf{1}_{A} \leqslant Y_{1}+\sum_{k=1}^{n-1}\left(Y_{k+1}-Y_{k}\right) \mathbf{1}_{D_{k}}-Y_{n} \mathbf{1}_{D_{n}}
$$

Proof. Introduce the events $E_{1}=\left\{Y_{1}>\varepsilon\right\}, E_{i}=\left\{Y_{1} \leqslant \varepsilon, \ldots, Y_{i-1} \leqslant \varepsilon, Y_{i}>\varepsilon\right\}, i=2$, $\ldots, n$. Obviously

$$
\varepsilon \mathbf{1}_{A}=\varepsilon \sum_{i=1}^{n} \mathbf{1}_{A E_{i}}=\sum_{i=1}^{n} \varepsilon \mathbf{1}_{E_{i}} \leqslant \sum_{i=1}^{n} Y_{i} \mathbf{1}_{E_{i}}
$$

Applying Abel's transformation, we can write

$$
\sum_{i=1}^{n} Y_{i} \mathbf{1}_{E_{i}}=\sum_{i=1}^{n-1} U_{i}\left(Y_{i}-Y_{i+1}\right)+U_{n} Y_{n}
$$

where

$$
U_{i}=\mathbf{1}_{E_{i}}+\ldots+\mathbf{1}_{E_{i}}=1-\mathbf{1}_{D 4_{1}}+\mathbf{1}_{D 4_{1}}-\mathbf{1}_{D_{2}}+\ldots+\mathbf{1}_{D_{i-1}}-\mathbf{1}_{D_{i}}=1-\mathbf{1}_{D_{i}} .
$$

Thus, by (4.2),

$$
\begin{aligned}
\sum_{i=1}^{n} Y_{i} \mathbf{1}_{E_{i}} & =\sum_{i=1}^{n-1}\left(1-\mathbf{1}_{D_{i}}\right)\left(Y_{i}-Y_{i+1}\right)+\left(1-\mathbf{1}_{D_{n}}\right) Y_{n} \\
& =Y_{1}+\sum_{i=1}^{n-1}\left(Y_{i+1}-Y_{i}\right) \mathbf{1}_{D_{i}}-Y_{n} \mathbf{1}_{D_{n}}
\end{aligned}
$$

Theorem 4.1. Let $X_{1}, \ldots, X_{n}$ be any random variables with finite second moments and $c_{1}, \ldots, c_{n}$ be any non-negative constants. Then

$$
\begin{aligned}
\varepsilon^{2} P\left\{\max _{m \leqslant k \leqslant n} c_{k}\left|\sum_{j=1}^{k} X_{j}\right|>\varepsilon\right\} \leqslant & c_{m}^{2} \sum_{i, j=1}^{m} \mathrm{E}\left(X_{i} X_{j}\right)+\sum_{k=m}^{n-1}\left|c_{k+1}^{2}-c_{k}^{2}\right| \sum_{i, j=1}^{k} \mathrm{E}\left(X_{i} X_{j}\right) \\
& +2 \sum_{k=m}^{n-1} c_{k+1}^{2} \mathrm{E}\left(\left|X_{k+1}\right|\left|\sum_{j=1}^{k} X_{j}\right|\right)+\sum_{k=m}^{n-1} c_{k+1}^{2} \mathrm{E} X_{k+1}^{2} .
\end{aligned}
$$

Proof. Applying (4.1) with $Y_{k}=c_{k}^{2}\left|\sum_{j=1}^{k} X_{j}\right|^{2}$, we obtain $\varepsilon^{2} P\left\{\max _{m \leqslant k \leqslant n} c_{k}^{2}\left|\sum_{j=1}^{k} X_{j}\right|^{2}>\varepsilon^{2}\right\} \leqslant c_{m}^{2} \mathrm{E}\left|\sum_{j=1}^{m} X_{j}\right|^{2}+\mathrm{E} \sum_{k=m}^{n-1}\left(c_{k+1}^{2}\left|\sum_{j=1}^{k+1} X_{j}\right|^{2}-c_{k}^{2}\left|\sum_{j=1}^{k} X_{j}\right|^{2}\right) \mathbf{1}_{D_{k}^{\prime}}$, 
where $D_{k}^{\prime}=\left\{Y_{m} \leqslant \varepsilon^{2}, \ldots, Y_{k} \leqslant \varepsilon^{2}\right\}$. Each summand on the right-hand side can be estimated as follows:

$$
\begin{aligned}
& \left(c_{k+1}^{2}\left|\sum_{j=1}^{k+1} X_{j}\right|^{2}-c_{k}^{2}\left|\sum_{j=1}^{k} X_{j}\right|^{2}\right) \mathbf{1}_{D_{k}^{\prime}} \\
& \quad \leqslant\left(c_{k+1}^{2}\left|\sum_{j=1}^{k} X_{j}\right|^{2}+2 c_{k+1}^{2}\left|\sum_{j=1}^{k} X_{j}\right|\left|X_{k+1}\right|+c_{k+1}^{2} X_{k+1}^{2}-c_{k}^{2}\left|\sum_{j=1}^{k} X_{j}\right|^{2}\right) \mathbf{1}_{D_{k}^{\prime}} \\
& \quad \leqslant\left|c_{k+1}^{2}-c_{k}^{2}\right|\left|\sum_{j=1}^{k} X_{j}\right|^{2}+2 c_{k+1}^{2}\left|\sum_{j=1}^{k} X_{j}\right|\left|X_{k+1}\right|+c_{k+1}^{2} X_{k+1}^{2},
\end{aligned}
$$

which yields (4.3).

\section{Consistency of the change-point estimator}

Assume that $\left\{r_{1, k}^{2}, k \in \mathbb{Z}\right\}$ and $\left\{r_{2, k}^{2}, k \in \mathbb{Z}\right\}$ are two $\operatorname{ARCH}(\infty)$ sequences defined by (3.2), where $a_{i} \geqslant 0$ and where $\left\{b_{i}(j)\right\}$ are sequences of non-negative numbers satisfying Assumption 3.1.

Suppose we observe a sample $r_{1}^{2}, \ldots, r_{n}^{2}$ from the model

$$
r_{k}^{2}= \begin{cases}r_{1, k}^{2}, & \text { if } 1 \leqslant k \leqslant k^{*}, \\ r_{2, k}^{2}, & \text { if } k^{*}<k \leqslant n,\end{cases}
$$

where $k^{*}$ is an unknown change-point. Let $k^{*}=\tau^{*} n$ and assume that $0<\tau^{*}<1$. Obviously, the 'disordered' sequence $\left\{r_{k}^{2}\right\}$ is no longer stationary. For example, for the ARCH(1) model such that $a_{1} \neq a_{2}$ and $b_{1}=b_{2}=b$ (cf. (3.25)), the covariance function has the form

$$
\operatorname{cov}\left(r_{k}^{2}, r_{k^{\prime}}^{2}\right)= \begin{cases}a_{1}^{2} K b^{\left|k^{\prime}-k\right|}, & \text { if } 1 \leqslant k, k^{\prime} \leqslant k^{*}, \\ a_{2}^{2} K b^{\left|k^{\prime}-k\right|}, & \text { if } k^{*}<k, k^{\prime} \leqslant n, \\ a_{1} \alpha_{2} K b^{\left|k^{\prime}-k\right|}, & \text { otherwise, }\end{cases}
$$

where $K$ is given by (3.24). Interestingly, the correlation function of the 'disordered' sequence is $b^{\left|k^{\prime}-k\right|}$ and does not reflect the existence of non-stationarity. Therefore in the following we focus on the covariance function.

For ease of reference we now state a simple consequence of Theorem 3.1 which follows from the relation $r_{i, k}^{2}=a_{i} Y_{k}$, with $Y_{k}$ given by (2.5).

Corollary 5.1. Suppose (3.2) and (5.1) hold and sequences $\left\{r_{1, k}^{2}\right\}$ and $\left\{r_{2, k}^{2}\right\}$ satisfy Assumption 3.1. Then 


$$
0 \leqslant \operatorname{cov}\left(r_{k}^{2}, r_{k^{\prime}}^{2}\right) \leqslant \begin{cases}a_{1}^{2} C_{1}^{\prime}(\alpha(1+\beta))^{\left|k^{\prime}-k\right|}, & \text { if } 1 \leqslant k, k^{\prime} \leqslant k^{*}, \\ a_{2}^{2} C_{1}^{\prime}(\alpha(1+\beta))^{\left|k^{\prime}-k\right|}, & \text { if } k^{*}<k, k^{\prime} \leqslant n \\ a_{1} a_{2} C_{1}^{\prime}(\alpha(1+\beta))^{\left|k^{\prime}-k\right|}, & \text { otherwise, }\end{cases}
$$

where

$$
C_{1}^{\prime}=\frac{\beta(\lambda-1)}{(1+\beta)(1-\alpha)(1-\bar{B})^{2}\left(1-\bar{B}^{2} \lambda\right)}
$$

and $\bar{B}=\max \left\{B_{1}, B_{2}\right\}$.

In the following, $C$ stands for a positive constant whose value may change from line to line. Consistency of the estimator $\hat{\tau}=\hat{k} / n$ defined by (1.3) follows from the following theorem.

Theorem 5.1. Consider a sample $r_{1}^{2}, \ldots, r_{n}^{2}$ satisfying (5.1), (3.2) and the change-point estimator $\hat{k}$ given by (1.3). If sequences $\left\{r_{1, k}^{2}\right\}$ and $\left\{r_{2, k}^{2}\right\}$ satisfy Assumption 3.1 and

$$
\Delta:=\frac{a_{1}}{1-B_{1}}-\frac{a_{2}}{1-B_{2}} \neq 0,
$$

then, for $\hat{\tau}=\hat{k} / n$,

$$
P\left\{\left|\hat{\tau}-\tau^{*}\right|>\varepsilon\right\} \leqslant \frac{C}{\varepsilon^{2} \Delta^{2}} n^{-1 / 2},
$$

where $C$ is some positive constant.

Proof. As a standard starting argument in the change-point problems, observe that $\left|\mathrm{E} R_{k}\right|$ reaches its maximum at the point $k^{*}$. Indeed, by the equality $\mathrm{E}_{i, k}^{2}=a_{i} /\left(1-B_{i}\right)$, which is a necessary condition for the stationarity of the sequence $\left\{r_{i, k}^{2}\right\}$,

$$
\mathrm{E} R_{k}= \begin{cases}\Delta \tau\left(1-\tau^{*}\right), & \text { if } k \leqslant k^{*} \\ \Delta \tau^{*}(1-\tau), & \text { if } k>k^{*}\end{cases}
$$

where $k=\tau n$. Thus

$$
\mathrm{E} R_{k^{*}}=\Delta \tau^{*}\left(1-\tau^{*}\right) .
$$

It follows from (5.4) and (5.5) that

$$
\left|\mathrm{E} R_{k^{*}}\right|-\left|\mathrm{E} R_{k}\right|= \begin{cases}|\Delta|\left(\tau^{*}-\tau\right)\left(1-\tau^{*}\right), & \text { if } k \leqslant k^{*}, \\ |\Delta|\left(\tau-\tau^{*}\right) \tau^{*}, & \text { if } k>k^{*},\end{cases}
$$

and hence

$$
\left|\mathrm{E} R_{k^{*}}\right|-\left|\mathrm{E} R_{k}\right| \geqslant\left|\Delta \| \tau^{*}-\tau\right|\left(\tau^{*} \wedge\left(1-\tau^{*}\right)\right) .
$$

Observe now that 


$$
\begin{aligned}
\left|R_{k}\right|-\left|R_{k^{*}}\right| & \leqslant\left|R_{k}-\mathrm{E} R_{k}\right|+\left|\mathrm{E} R_{k}\right|+\left|R_{k^{*}}-\mathrm{E} R_{k^{*}}\right|-\left|\mathrm{E} R_{k^{*}}\right| \\
& \leqslant 2 \max _{1 \leqslant k \leqslant n}\left|R_{k}-\mathrm{E} R_{k}\right|+\left|\mathrm{E} R_{k}\right|-\left|\mathrm{E} R_{k^{*}}\right|,
\end{aligned}
$$

and thus, by (5.6),

$$
\begin{aligned}
|\Delta|\left|\tau^{*}-\tau\right|\left(\tau^{*} \wedge\left(1-\tau^{*}\right)\right) & \leqslant\left|\mathrm{E} R_{k^{*}}\right|-\left|\mathrm{E} R_{k}\right| \\
& \leqslant 2 \max _{1 \leqslant k \leqslant n}\left|R_{k}-\mathrm{E} R_{k}\right|+\left|R_{k^{*}}\right|-\left|R_{k}\right| .
\end{aligned}
$$

Replacing $\tau$ by $\hat{\tau}$ in (5.7) and noting that $\left|R_{k^{*}}\right| \leqslant\left|R_{\hat{k}}\right|$, we obtain

$$
|\Delta|\left|\tau^{*}-\hat{\tau}\right|\left(\tau^{*} \wedge\left(1-\tau^{*}\right)\right) \leqslant 2 \max _{1 \leqslant k \leqslant n}\left|R_{k}-\mathrm{E} R_{k}\right| .
$$

It now remains to estimate $\max _{1 \leqslant k \leqslant n}\left|R_{k}-\mathrm{E} R_{k}\right|$. Observing that

$$
\begin{aligned}
\left|R_{k}-\mathrm{E} R_{k}\right| & =\frac{1}{n^{2}}\left|n \sum_{j=1}^{k}\left(r_{j}^{2}-\mathrm{E} r_{j}^{2}\right)-k \sum_{j=1}^{n}\left(r_{j}^{2}-\mathrm{E} r_{j}^{2}\right)\right| \\
& \leqslant \frac{1}{n}\left|\sum_{j=1}^{k}\left(r_{j}^{2}-\mathrm{E} r_{j}^{2}\right)\right|+\frac{1}{n}\left|\sum_{j=1}^{n}\left(r_{j}^{2}-\mathrm{E} r_{j}^{2}\right)\right|
\end{aligned}
$$

we obtain

$$
\max _{1 \leqslant k \leqslant n}\left|R_{k}-\mathrm{E} R_{k}\right| \leqslant 2 \max _{1 \leqslant k \leqslant n} \frac{1}{n}\left|\sum_{j=1}^{k}\left(r_{j}^{2} \mathrm{E} r_{j}^{2}\right)\right| .
$$

Applying Theorem 4.1 with $m=1, X_{k}=r_{k}^{2}-\mathrm{E} r_{k}^{2}$ and $c_{1}=\ldots=c_{n}=1 / n$, we obtain

$$
\begin{aligned}
& \varepsilon^{2} P\left\{\max _{1 \leqslant k \leqslant n} \frac{1}{n}\left|\sum_{j=1}^{k}\left(r_{j}^{2}-\mathrm{E} r_{j}^{2}\right)\right|>\varepsilon\right\} \\
& \leqslant \frac{2}{n^{2}} \sum_{k=1}^{n-1} \mathrm{E}\left(\left|r_{k+1}^{2}-\mathrm{E}_{k+1}^{2}\right|\left|\sum_{j=1}^{k}\left(r_{j}^{2}-\mathrm{E} r_{j}^{2}\right)\right|\right)+\frac{1}{n^{2}} \sum_{k=0}^{n-1} \mathrm{E}\left(r_{k+1}^{2}-\mathrm{E} r_{k+1}^{2}\right)^{2} . \\
& \leqslant \frac{2}{n^{2}} \sum_{k=1}^{n-1}\left(\operatorname{var} r_{k+1}^{2}\right)^{1 / 2}\left(\sum_{j, j^{\prime}=1}^{k} \operatorname{cov}\left(r_{j}^{2}, r_{j^{\prime}}^{2}\right)\right)^{1 / 2}+\frac{1}{n^{2}} \sum_{k=0}^{n-1} \operatorname{var} r_{k+1}^{2} .
\end{aligned}
$$

By Corollary 5.1,

$$
\operatorname{cov}\left(r_{j}^{2}, r_{j^{\prime}}^{2}\right) \leqslant \Lambda((1+\beta) \alpha)^{\left|j-j^{\prime}\right|},
$$

where $\Lambda=C_{1}^{\prime} \max \left\{a_{1}^{2}, a_{2}^{2}, a_{1} a_{2}\right\}$. Denoting $\max \left\{\operatorname{var} r_{1,0}^{2}, \operatorname{var} r_{2,0}^{2}\right\}=: S^{2}$, it follows that 


$$
\begin{aligned}
\varepsilon^{2} P\left\{\max _{1 \leqslant k \leqslant n} \frac{1}{n}\left|\sum_{j=1}^{k}\left(r_{j}^{2}-\mathrm{E} r_{j}^{2}\right)\right|>\varepsilon\right\} & \leqslant \frac{2 S \sqrt{\Lambda}}{n^{2}} \sum_{k=1}^{n-1}\left(\sum_{j, j^{\prime}=1}^{k}((1+\beta) \alpha)^{\left|j-j^{\prime}\right|}\right)^{1 / 2}+\frac{1}{n} S^{2} \\
& \leqslant \frac{K_{1}}{n^{2}} \sum_{k=1}^{n-1} k^{1 / 2}+\frac{1}{n} S^{2} \leqslant K_{2} n^{-1 / 2},
\end{aligned}
$$

where $K_{1}$ and $K_{2}$ are positive constants.

Combining (5.8), (5.9) and (5.11) yields (5.3).

Consider now the change-point model (3.25) where the ARCH(1) sequences start from $k=0$ rather than from $-\infty$.

Theorem 5.2. Consider the sample $r_{1}^{2}, \ldots, r_{n}^{2}$ satisfying (5.1), (3.25) and the change-point estimator $\hat{k}$ defined by (1.3). Then, for $\hat{\tau}=\hat{k} / n$,

$$
P\left\{\left|\hat{\tau}-\tau^{*}\right|>\varepsilon\right\} \leqslant \frac{C}{\varepsilon^{2} \Delta^{2}} n^{-1 / 2} .
$$

Proof. Denote $b:=\max \left\{b_{1}, b_{2}\right\}$. Using Theorem 3.2, we obtain, similarly to the proof of Theorem 5.1,

$$
\varepsilon^{2} P\left\{\max _{1 \leqslant k \leqslant n} \frac{1}{n}\left|\sum_{j=1}^{k}\left(r_{j}^{2}-\mathrm{E} r_{j}^{2}\right)\right|>\varepsilon\right\} \leqslant \frac{2 S}{n^{2}} \sum_{k=1}^{n-1}\left(\sum_{j, j^{\prime}=1}^{k}\left(U b^{\left|j-j^{\prime}\right|}+|V| b^{j+j^{\prime}} \lambda^{j \wedge j^{\prime}}\right)\right)^{1 / 2}+\frac{1}{n} S^{2}
$$

where $U$ and $V$ are defined in (3.27). The summands under the square root can be estimated as follows:

$$
\begin{aligned}
\sum_{j, j^{\prime}=1}^{k}\left(U b^{\left|j-j^{\prime}\right|}+|V| b^{j+j^{\prime}} \lambda^{j \wedge j^{\prime}}\right) & \leqslant U \sum_{|j|<k}(k-|j|) b^{|j|}+|V| \sum_{j=1}^{k}\left(b^{2} \lambda\right)^{j}+2|V| \sum_{j=1}^{k} \sum_{j^{\prime}=1}^{j}\left(b^{2} \lambda\right)^{j} \\
& \leqslant U k \sum_{|j|<k} b^{|j|}+|V| \frac{b^{2} \lambda}{1-b^{2} \lambda}+2|V| k \frac{b^{2} \lambda}{1-b^{2} \lambda} \leqslant K_{3} k
\end{aligned}
$$

This, together with (5.13) and (5.8), yields (5.12).

Using inequality (5.8) it is easy to verify that the estimator $\hat{\tau}$ is still consistent when the difference $\Delta=\Delta(n)$ defined by (5.2) tends to zero, as $n \rightarrow \infty$, at a rate slower that $n^{-1 / 4}$. Suppose that

$$
r_{k}^{2}=\left(r_{k}^{(n)}\right)^{2}= \begin{cases}\left(r_{1, k}^{(n)}\right)^{2}, & \text { if } 1 \leqslant k \leqslant k^{*} \\ \left(r_{2, k}^{(n)}\right)^{2}, & \text { if } k^{*}<k \leqslant n\end{cases}
$$


where $k^{*}=\left[n \tau^{*}\right], \tau^{*} \in(0,1)$ and $\left(r_{i, k}^{(n)}\right)^{2}, i=1,2$, are given by equations

$$
\left(r_{i, k}^{(n)}\right)^{2}=\left(\sigma_{i, k}^{(n)}\right)^{2} \varepsilon_{k}^{2}, \quad\left(\sigma_{i, k}^{(n)}\right)^{2}=a_{i}^{(n)}+\sum_{j=1}^{\infty} b_{i}^{(n)}(j)\left(r_{i, k-j}^{(n)}\right)^{2} .
$$

Denote $B_{i}^{(n)}:=b_{i}^{(n)}(1)+b_{i}^{(n)}(2)+\ldots$ Then we have the following corollary.

Corollary 5.2. Let the sample $r_{1}^{2}, \ldots, r_{n}^{2}$ be given by (5.14) and (5.15), and let sequences $\left\{r_{1, k}^{(n) 2}\right\}$ and $\left\{r_{2, k}^{(n) 2}\right\}$ satisfy Assumption 3.1 (with fixed $\alpha$ and $\beta$ ) for any $n \geqslant 1$. Assume that $a_{1}^{(n)}, a_{2}^{(n)}$ are bounded sequences and

$$
\Delta(n):=a_{2}^{(n)} /\left(1-B_{2}^{(n)}\right)-a_{1}^{(n)} /\left(1-B_{1}^{(n)}\right)
$$

satisfies $\Delta(n) \rightarrow 0$ and $\Delta^{4}(n) n \rightarrow \infty$ as $n \rightarrow \infty$. Then $\hat{\tau}$ is a weakly consistent estimator of $\tau^{*}$.

The same result holds in the case of $\operatorname{ARCH}(1)$ sequences (3.25).

\section{Rate of convergence}

In Section 5 the consistency of the estimator was established. Using this result, we will show in the present section that $\left|\hat{\tau}-\tau^{*}\right|=O_{P}(1 / n)$, that is,

$$
\lim _{M \rightarrow \infty} \sup _{n} P\left\{\left|\hat{\tau}-\tau^{*}\right|>M / n\right\}=0 .
$$

Relation (6.1) shows that the estimator $\hat{\tau}$ converges at the same rate as the least-squares estimator studied by Bai (1994) in the case of weakly dependent linear sequences. The general idea of our proof is similar to Bai's proof, but given a different form of the HájekRényi inequality, there are some differences. We have also been able to avoid a number of technical arguments used by Bai.

Theorem 6.1. Under the assumptions of Theorem 5.1.

$$
\left|\hat{\tau}-\tau^{*}\right|=O_{P}\left(\frac{1}{n}\right)
$$

(Recall that $\tau^{*} \in(0,1)$ is fixed and $k^{*}=\left[n \tau^{*}\right]$.)

Proof. Observe that 


$$
\begin{aligned}
\left\{\left|\hat{\tau}-\tau^{*}\right|>M / n\right\} & \subset\left\{\hat{k}-k^{*}>M\right\} \cup\left\{\hat{k}-k^{*}<-(M-1)\right\} \\
& \subset\left\{\max _{k \leqslant k^{*}+M}\left|R_{k}\right|<\max _{1 \leqslant k \leqslant n}\left|R_{k}\right|\right\} \cup\left\{\max _{k \leqslant k^{*}-(M-1)}\left|R_{k}\right|=\max _{1 \leqslant k \leqslant n}\left|R_{k}\right|\right\} \\
& \subset\left\{\max _{k \geqslant k^{*}+M}\left|R_{k}\right|=\max _{1 \leqslant k \leqslant n}\left|R_{k}\right|\right\} \cup\left\{\max _{k \leqslant k^{*}-(M-1)}\left|R_{k}\right|=\max _{1 \leqslant k \leqslant n}\left|R_{k}\right|\right\} \\
& \subset\left\{\max _{k \geqslant k^{*}+M}\left|R_{k}\right| \geqslant\left|R_{k^{*}}\right|\right\} \cup\left\{\max _{k \leqslant k^{*}-(M-1)}\left|R_{k}\right| \geqslant\left|R_{k^{*}}\right|\right\} .
\end{aligned}
$$

Thus

$$
P\left\{\left|\hat{\tau}-\tau^{*}\right|>M / n\right\} \leqslant P\left\{\max _{k \geqslant k^{*}+M}\left|R_{k}\right| \geqslant\left|R_{k^{*}}\right|\right\}+P\left\{\max _{k \leqslant k^{*}-(M-1)}\left|R_{k}\right| \geqslant\left|R_{k^{*}}\right|\right\} .
$$

Because of symmetry, we focus only on the first term. Since $\{|x| \geqslant|y|\}=\{x-y \geqslant 0$, $x+y \geqslant 0\} \cup\{x-y \leqslant 0, x+y \leqslant 0\}$, we have

$$
P\left\{\max _{k \geqslant k^{*}+M}\left|R_{k}\right| \geqslant\left|R_{k^{*}}\right|\right\} \leqslant P\left\{\max _{k \geqslant k^{*}+M} R_{k}-R_{k^{*}} \geqslant 0\right\}+P\left\{\min _{k \geqslant k^{*}+M} R_{k}+R_{k^{*}} \leqslant 0\right\}=: i_{1}+i_{2} .
$$

We will show that $\lim _{M \rightarrow \infty} \sup _{n} i_{1}=0$ and $\lim _{M \rightarrow \infty} \sup _{n} i_{2}=0$. Without loss of generality, assume that $\Delta>0$, so that $\mathrm{E} R_{k} \geqslant 0$ (see (5.4)).

We consider only $i_{1}$. The term $i_{2}$ is dealt with analogously. We have

$$
\left\{R_{k}-R_{k^{*}} \geqslant 0\right\}=\left\{R_{k}-\mathrm{E} R_{k}-R_{k^{*}}+\mathrm{E} R_{k^{*}} \geqslant \mathrm{E} R_{k^{*}}-\mathrm{E} R_{k}\right\} .
$$

Observe that

$$
R_{k}-\mathrm{E} R_{k}-R_{k^{*}}+\mathrm{E} R_{k^{*}}=n^{-2}\left(n \sum_{j=k^{*}+1}^{k}\left(r_{j}^{2}-\mathrm{E} r_{j}^{2}\right)-\left(k-k^{*}\right) \sum_{j=1}^{n}\left(r_{j}^{2}-\mathrm{E} r_{j}^{2}\right)\right)
$$

Thus

$$
\left\{R_{k}-R_{k^{*}} \geqslant 0\right\} \subset\left\{n^{-1}\left|\sum_{j=k^{*}+1}^{k}\left(r_{j}^{2}-\mathrm{E} r_{j}^{2}\right)\right|+n^{-2}\left(k-k^{*}\right)\left|\sum_{j=1}^{n}\left(r_{j}^{2}-\mathrm{E} r_{j}^{2}\right)\right| \geqslant \mathrm{E} R_{k^{*}}-\mathrm{E} R_{k}\right\} .
$$

We know from (5.6) (recall that we assume $\Delta>0$ so that $\mathrm{E} R k^{*} \geqslant 0, \mathrm{E} R_{k} \geqslant 0$ ) that for some $C>0$

$$
\mathrm{E} R_{k^{*}}-\mathrm{E} R_{k} \geqslant C \Delta \frac{\left|k^{*}-k\right|}{n},
$$

so that 


$$
\begin{aligned}
i_{1}= & P\left\{\max _{k \geqslant k^{*}+M} R_{k}-R_{k^{*}} \geqslant 0\right\} \\
\leqslant & P\left\{\max _{k \geqslant k^{*}+M}\left(k-k^{*}\right)^{-1}\left|\sum_{j=k^{*}+1}^{k}\left(r_{j}^{2}-\mathrm{E} r_{j}^{2}\right)\right| \geqslant C \Delta / 2\right\} \\
& +P\left\{n^{-1}\left|\sum_{j=1}^{n}\left(r_{j}^{2}-\mathrm{E} r_{j}^{2}\right)\right| \geqslant C \Delta / 2\right\} \\
= & : j_{1}+j_{2} .
\end{aligned}
$$

Obviously

$$
P\left\{n^{-1}\left|\sum_{j=1}^{n}\left(r_{j}^{2}-\mathrm{E} r_{j}^{2}\right)\right| \geqslant C \Delta / 2\right\} \leqslant \frac{\tilde{C}}{\Delta^{2}} n^{-1 / 2}
$$

for some $\tilde{C}>0$, so that $j_{2} \rightarrow 0$ as $n \rightarrow \infty$.

To prove that $j_{1} \rightarrow 0$ we use Theorem 3.1 and Theorem 4.1 with $c_{k}=1 / k$ and $X_{j}=r_{j+p}^{2}-\mathrm{E} r_{j+p}^{2}$, where $p$ is an arbitrary integer. The third term on the right-hand side of (4.3) is then of order $m^{-1 / 2}$, whereas the remaining terms are of order $m^{-1}$. So we obtain

$$
P\left\{\max _{m \leqslant k \leqslant n} \frac{1}{k}\left|\sum_{j=1}^{k}\left(r_{j+p}^{2}-\mathrm{E} r_{j+p}^{2}\right)\right|>\varepsilon\right\} \leqslant K \varepsilon^{-2} m^{-1 / 2},
$$

for some $K>0$. Thus, by (6.2),

$$
j_{1} \leqslant P\left\{\max _{l \geqslant M} \frac{1}{l}\left|\sum_{i=1}^{l}\left(r_{i+k^{*}}^{2}-\mathrm{E}_{i+k^{*}}^{2}\right)\right| \geqslant C \Delta / 2\right\} \leqslant \frac{4 K}{C^{2} \Delta^{2}} M^{-1 / 2} \rightarrow 0,
$$

as $M \rightarrow \infty$.

Remark 6.1. Replacing the inequality $k \geqslant k^{*}+M$ by $k \geqslant k^{*}+M / \Delta^{4}$ in the proof of Theorem 6.1 , it can be easily verified that

$$
\left|\hat{\tau}-\tau^{*}\right|=O_{P}\left(\frac{1}{n \Delta^{4}}\right),
$$

where $\Delta=\Delta(n)$ also depends on $n$.

\section{Simulations and a data example}

In this section we briefly discuss the sampling distribution of the change-point estimator $\hat{k}$ and then apply it to two financial time series.

The estimator $\hat{k}$ shares most properties of analogous CUSUM type estimators; see, for example, Antoch et al. (1997) and Horváth and Kokoszka (1997). It is skewed to the left, 
and the degree of skewness increases as the change-point $k^{*}$ moves to the right. Estimates are most accurate if $k^{*}$ lies around the middle of the sample. Obviously, the accuracy increases with the sample size and the size of the difference of variances before and after $k^{*}$. Interestingly, it appears that the estimator $\hat{k}$ has larger spread when the change in variance is due to the change in the $b(j)$ rather than $a$. All these properties are illustrated by the histograms in Figures 2-4. Each histogram describes the sample distribution of 1000 replications of $\hat{k}$ for several ARCH(1) models. Series of length $n=10^{3}$ were estimated. In Figures 2 and 4, $k^{*}=500$; in Figure 3, $k^{*}=750$. In all the figures, $a_{1}=1$ and $b(1)=0.1$. In Figures 2(a), 3(a) and 4(a) the variance increases by 30\%; in Figures 2(b), 3(b) and 4(b) it increases by $90 \%$.

We conclude this section by applying our method to series of returns on foreign exchange rates. If $P_{t}$ is the price of a currency in US dollars on day $t$, the return is defined

(a)

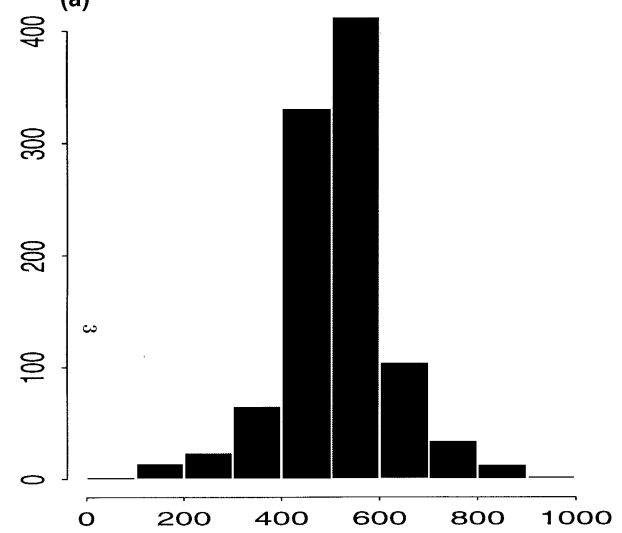

(b)

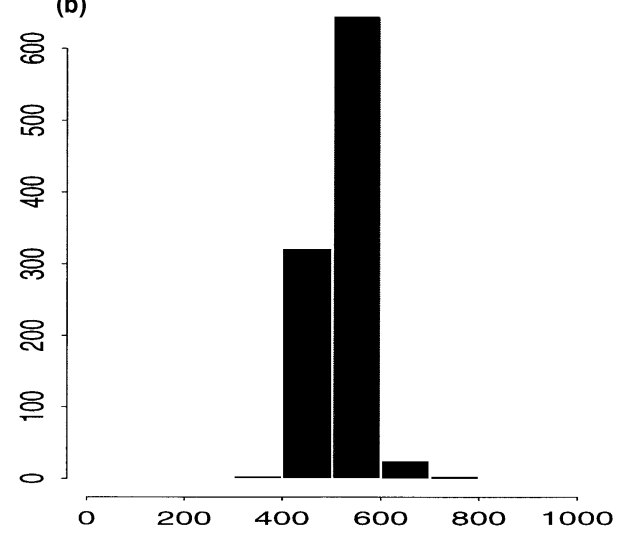

Figure 2. Sampling distribution of $\hat{k}, b(1)=b(2)=0.1$. (a) $a_{2}=1.3$; (b) $a_{2}=1.8$.

(a)

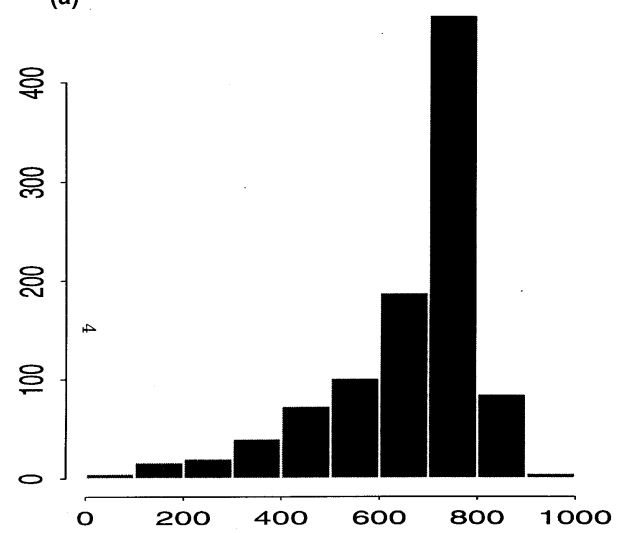

(b)

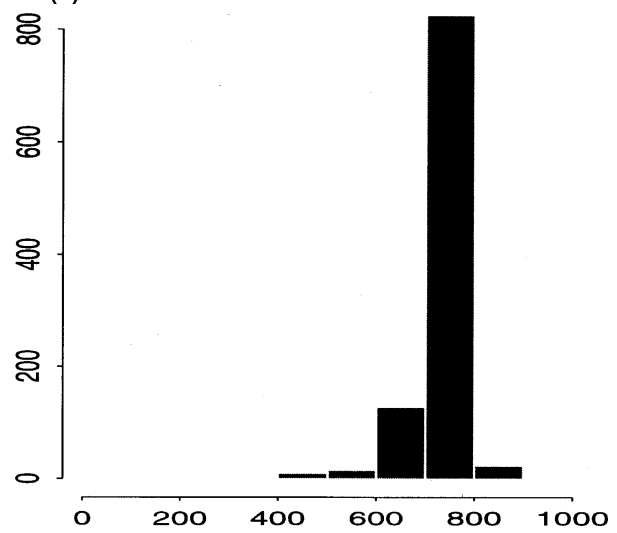

Figure 3. Sampling distribution of $\hat{k}, b(1)=b(2)=0.1$. (a) $a_{2}=1.3$; (b) $a_{2}=1.8$. 
(a)

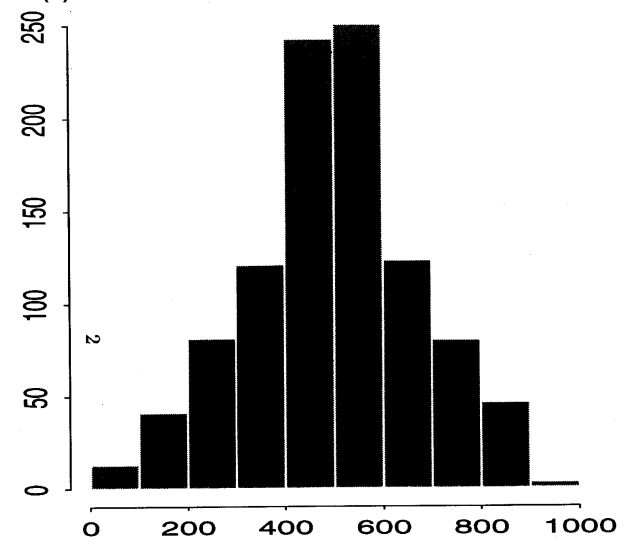

(b)

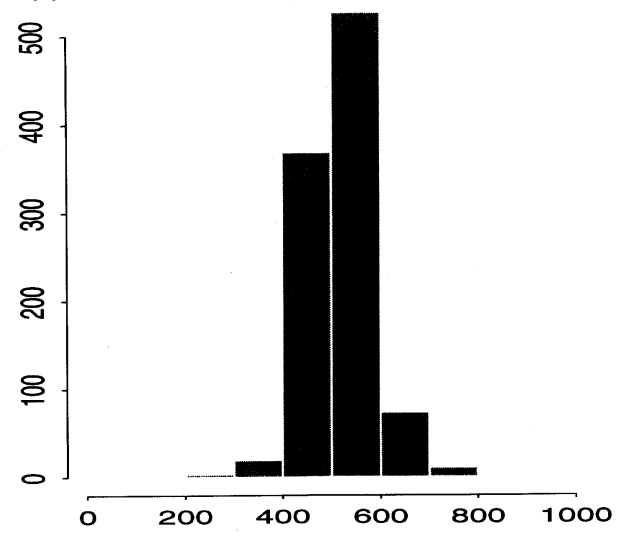

Figure 4. Sampling distribution of $\hat{k}$, (a) $a_{2}=1.25, b(2)=0.013$. (b) $a_{2}=1.6, b(2)=0.02$.

as $r_{t}=\log P_{t}-\log P_{t-1}$. Figure 5(a) shows 6630 daily returns on the DM exchange rate from 24 August 1971 to 21 January 1997. Recall that 24 August 1971 marks the end of the Bretton Woods fixed exchange rates system. Figure 5(b) shows the sequences $R_{k}$ for returns on DM and pound sterling exchange rates. The peak around $k=2100$ corresponds to 1979 , when the European Monetary System (EMS) was introduced. Interestingly, even though the
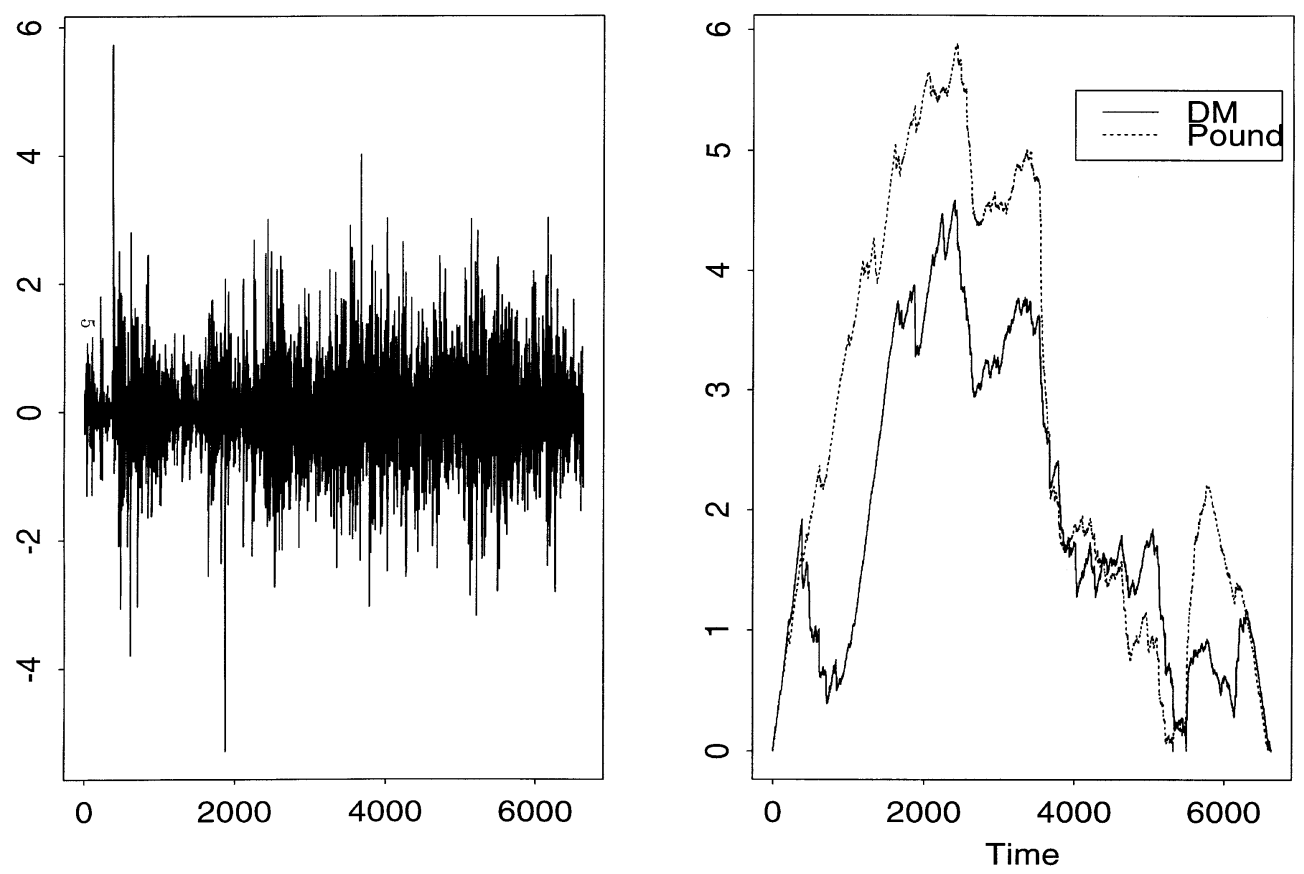

Figure 5. Analysis of returns on exchange rates. 
United Kingdom joined the EMS in October 1990 and left two years afterwards, the impact of the system on the volatility of the pound is similar to that on the DM.

\section{Appendix: Proof of Theorem 3.2}

Applying recursively equations (3.25), we obtain, for $i=1,2$,

$$
r_{i, k}^{2}=a_{i} \sum_{j=0}^{k-1} b_{i}^{j} \prod_{m=0}^{j} \varepsilon_{k-m}^{2}+b_{i}^{k} r_{i, 0}^{2} \prod_{m=0}^{k-1} \varepsilon_{k-m}^{2} .
$$

Without loss of generality, suppose that $k \leqslant k^{\prime}$. Then, multiplying $r_{1, k}^{2}$ and $r_{2, k^{\prime}}^{2}$, due to (A.1), we can write $\mathrm{E} r_{1, k}^{2} r_{2, k^{\prime}}^{2}$ as

$$
\mathrm{E} r_{1, k}^{2} r_{2, k^{\prime}}^{2}=I_{1}+I_{2}+I_{3}+I_{4}
$$

where

$$
\begin{aligned}
& I_{1}:=a_{1} a_{2} \sum_{j=0}^{k-1} \sum_{j^{\prime}=0}^{k^{\prime}-1} b_{1}^{j} b_{2}^{j^{\prime}} \mathrm{E} \prod_{m=0}^{j} \varepsilon_{k-m}^{2} \prod_{m^{\prime}=0}^{j^{\prime}} \varepsilon_{k^{\prime}-m^{\prime}}^{2}, \\
& I_{2}:=a_{1} b_{2}^{k^{\prime}} \mathrm{E} r_{2,0}^{2} \sum_{j=0}^{k-1} b_{1}^{j} \mathrm{E} \prod_{m=0}^{j} \varepsilon_{k-m}^{2} \prod_{m^{\prime}=0}^{k^{\prime}-1} \varepsilon_{k^{\prime}-m^{\prime}}^{2}, \\
& I_{3}:=a_{2} b_{1}^{k} \mathrm{E} r_{1,0}^{2} \sum_{j=0}^{k^{\prime}-1} b_{2}^{j^{\prime}} \mathrm{E} \prod_{m^{\prime}=0}^{j^{\prime}} \varepsilon_{k^{\prime}-m^{\prime}}^{2} \prod_{m=0}^{k-1} \varepsilon_{k-m}^{2}, \\
& I_{4}:=b_{1}^{k} b_{2}^{k^{\prime}} \mathrm{E}\left(r_{1,0}^{2} r_{2,0}^{2}\right) \mathrm{E} \prod_{m=0}^{k-1} \varepsilon_{k-m}^{2} \prod_{m^{\prime}=0}^{k^{\prime}-1} \varepsilon_{k^{\prime}-m^{\prime}}^{2} .
\end{aligned}
$$

$I_{1}$ can be split into two terms, the first of which has two independent products:

$$
\begin{aligned}
I_{1} & :=a_{1} a_{2} \sum_{j=0}^{k-1} \sum_{j^{\prime}=0}^{k^{\prime}-k-1} b_{1}^{j} b_{2}^{j^{\prime}} \mathrm{E} \prod_{m=0}^{j} \varepsilon_{k-m}^{2} \prod_{m^{\prime}=0}^{j^{\prime}} \varepsilon_{k^{\prime}-m^{\prime}}^{2}+a_{1} a_{2} \sum_{j=0}^{k-1} \sum_{j^{\prime}=k^{\prime}-k}^{k^{\prime}-1} b_{1}^{j} b_{2}^{j^{\prime}} \mathrm{E} \prod_{m=0}^{j} \varepsilon_{k-m}^{2} \prod_{m^{\prime}=0}^{j^{\prime}} \varepsilon_{k^{\prime}-m^{\prime}}^{2} \\
& =: I_{11}+I_{12} .
\end{aligned}
$$

Here

$$
\begin{aligned}
I_{11} & =a_{1} a_{2} \sum_{j=0}^{k-1} \sum_{j^{\prime}=0}^{k^{\prime}-k-1} b_{1}^{j} b_{2}^{j^{\prime}}=a_{1} a_{2} \frac{\left(1-b_{1}^{k}\right)\left(1-b_{2}^{k^{\prime}-k}\right)}{\left(1-b_{1}\right)\left(1-b_{2}\right)} \\
& =c_{1}-c_{1} b_{1}^{k}+c_{1} b_{1}^{k} b_{2}^{k^{\prime}-k}-c_{1} b_{2}^{k^{\prime}-k}
\end{aligned}
$$

where 


$$
c_{1}=\frac{a_{1} a_{2}}{\left(1-b_{1}\right)\left(1-b_{2}\right)} .
$$

Suppose that $b_{1} \lambda \neq 1$ and $b_{2} \lambda \neq 1$. The term $I_{12}$, after straightforward algebra, can be written as

$$
\begin{aligned}
I_{12} & =a_{1} a_{2} \lambda \sum_{j=1}^{k} b_{2}^{k^{\prime}-j}\left(1+b_{1} \lambda+\ldots+\left(b_{1} \lambda\right)^{k-j-1}+\left(b_{1} \lambda\right)^{k-j}\left(1+b_{1}+\ldots+b_{1}^{j-1}\right)\right) \\
& =a_{1} a_{2} \lambda \sum_{j=1}^{k} b_{2}^{k^{\prime}-j}\left(\frac{1-\left(b_{1} \lambda\right)^{k-j}}{1-b_{1} \lambda}+\left(b_{1} \lambda\right)^{k-j} \frac{1-b_{1}^{j}}{1-b_{1}}\right) \\
& =c_{2} b_{2}^{k^{\prime}-k}+c_{3}\left(b_{1} \lambda\right)^{k} b_{2}^{k^{\prime}}+c_{4} b_{2}^{k^{\prime}}+c_{5} b_{1}^{k} b_{2}^{k^{\prime}-k},
\end{aligned}
$$

where the constants $c_{2}, c_{3}, c_{4}$ and $c_{5}$ are

$$
\begin{aligned}
& c_{2}=a_{1} a_{2}\left(\frac{\lambda}{\left(1-b_{2}\right)\left(1-b_{1} \lambda\right)}-\frac{\lambda}{\left(1-b_{1} \lambda\right)\left(1-b_{1} b_{2} \lambda\right)}+\frac{\lambda}{\left(1-b_{1}\right)\left(1-b_{1} b_{2} \lambda\right)}\right), \\
& c_{3}=a_{1} a_{2}\left(\frac{\lambda}{\left(1-b_{1} \lambda\right)\left(1-b_{1} b_{2} \lambda\right)}-\frac{\lambda}{\left(1-b_{1}\right)\left(1-b_{1} b_{2} \lambda\right)}+\frac{\lambda}{\left(1-b_{1}\right)\left(1-b_{2} \lambda\right)}\right), \\
& c_{4}=-a_{1} a_{2} \frac{\lambda}{\left(1-b_{2}\right)\left(1-b_{1} \lambda\right)}, \\
& c_{5}=-a_{1} a_{2} \frac{\lambda}{\left(1-b_{1}\right)\left(1-b_{2} \lambda\right)},
\end{aligned}
$$

For the term $I_{2}$ we have:

$$
I_{2}=a_{1} b_{2}^{k^{\prime}} \mathrm{E} r_{2,0}^{2} \sum_{j=0}^{k-1} b_{1}^{j} \lambda^{j+1}=-c_{4} b_{2}^{k^{\prime}}+c_{4}\left(b_{1} \lambda\right)^{k} b_{2}^{k^{\prime}} .
$$

Similarly to the case of the term $I_{12}$, we obtain that

$$
\begin{aligned}
I_{3} & =a_{2} b_{1}^{k} \operatorname{Er}_{1,0}^{2}\left(1+b_{2}+\ldots+b_{2}^{k^{\prime}-k-1}+b_{2}^{k^{\prime}-k} \lambda\left(1+b_{2} \lambda+\ldots+\left(b_{2} \lambda\right)^{k-1}\right)\right) \\
& =a_{2} b_{1}^{k} \operatorname{Er}_{1,0}^{2}\left(\frac{1-b_{2}^{k^{\prime}-k}}{1-b_{2}}+b_{2}^{k^{\prime}-k} \lambda \frac{1-\left(b_{2} \lambda\right)^{k}}{1-b_{2} \lambda}\right) \\
& =c_{1} b_{1}^{k}+c_{6} b_{1}^{k} b_{2}^{k^{\prime}-k}+c_{5}\left(b_{1} \lambda\right)^{k} b_{2}^{k^{\prime}},
\end{aligned}
$$

where

$$
c_{6}=a_{1} a_{2}\left(-\frac{1}{\left(1-b_{1}\right)\left(1-b_{2}\right)}+\frac{\lambda}{\left(1-b_{1}\right)\left(1-b_{2} \lambda\right)}\right)=-c_{1}-c_{5} .
$$

Finally 


$$
I_{4}=\mathrm{E}\left(r_{1,0}^{2} r_{2,0}^{2}\right)\left(b_{1} \lambda\right)^{k} b_{2}^{k^{\prime}} .
$$

Gathering the corresponding coefficients in the sum $I_{11}+I_{12}+I_{2}+I_{3}+I_{4}$, we see that

$$
\begin{aligned}
\mathrm{E}\left(r_{1, k}^{2} r_{2, k^{\prime}}^{2}\right)= & \frac{a_{1} a_{2}}{\left(1-b_{1}\right)\left(1-b_{2}\right)}+\left(-c_{1}+c_{1}\right) b_{1}^{k}+\left(c_{4}-c_{4}\right) b_{2}^{k^{\prime}}+\left(c_{1}+c_{5}+c_{6}\right) b_{1}^{k} b_{2}^{k^{\prime}-k} \\
& +\left(-c_{1}+c_{2}\right) b_{2}^{k^{\prime}-k}+\left(c_{3}+c_{4}+c_{5}\right)\left(b_{1} \lambda\right)^{k} b_{2}^{k^{\prime}} \\
= & \frac{a_{1} a_{2}}{\left(1-b_{1}\right)\left(1-b_{2}\right)}+U b_{2}^{k^{\prime}-k}+V\left(b_{1} \lambda\right)^{k} b_{2}^{k^{\prime}} .
\end{aligned}
$$

Now applying the equality

$$
\mathrm{E} r_{1, k}^{2} \mathrm{E} r_{2, k^{\prime}}^{2}=\frac{a_{1} a_{2}}{\left(1-b_{1}\right)\left(1-b_{2}\right)},
$$

we obtain the statement of theorem in the case where $b_{1} \lambda \neq 1$ and $b_{2} \lambda \neq 1$. The claim in the case $b_{1} \lambda=1$ or $b_{2} \lambda=1$ can be verified easily.

\section{Acknowledgements}

We would like to thank Paul Embrechts for pointing out the importance of the problem studied in this paper, Lajos Horváth for his careful reading of the manuscript and indicating several relevant references, and an anonymous referee for encouraging us to undertake the research presented in Section 6. The exchange rates data used in Section 7 were made available by Gilles Teyssière.

This research was partially supported by EPSRC grant GR/L/78222 at the University of Liverpool. Piotr Kokoszka was also partially supported by a NATO grant CRG 960503 and Remigijus Leipus was partially supported by Lithuanian State Science and Studies Foundation Grant K-014.

\section{References}

Antoch, J., Hušková, M. and Prášková, Z. (1997) Effect of dependence on statistics for determination of change. J. Statist. Plann. Inference, 60, 291-310.

Bai, J. (1994) Least squares estimation of a shift in linear processes. J. Time Ser. Anal. 15, 453-472.

Bai, J. and Perron, P. (1998) Estimating and testing linear models with multiple structural changes. Econometrica, 66, 47-78.

Bollerslev, T. (1986) Generalized autoregressive conditional heteroskedasticity. J. Econometrics, 31, 307-327.

Bollerslev, T. (1988) On the correlation structure for the generalized autoregressive conditional heteroskedastic process. J. Time Ser. Anal., 9, 121-131.

Bollerslev, T., Chou, R.Y. and Kroner, K.F. (1992) ARCH modeling in finance: a review of the theory and empirical evidence. J. Econometrics, 52, 5-59.

Brodsky, B.E. and Darkhovsky, B.S. (1993) Nonparametric Methods in Change-Point Problems. 
Dordrecht: Kluwer.

Chen, J. and Gupta, A.K. (1997) Testing and locating variance changepoints with application to stock prices. J. Amer. Statist. Assoc., 92, 739-747.

Csörgö, M. and Horváth, L. (1988) Nonparametric methods for change point problems. In P.R. Krishnaiah and C.R. Rao (eds), Handbook of Statistics, Vol. 7, Quality Control and Reliability, pp. 403-425. Amsterdam: North Holland.

Csörgő, M. and Horváth, L. (1997) Limited Theorems in Change-Point Analysis. Chichester: Wiley.

Davis, R.A., Huang, D. and Yao, Y.C. (1995) Testing for a change in the parameter values and order of an autoregressive model. Ann. Statist. 23, 282-304.

Embrechts, P., Klüppelberg, C. and Mikosch, T. (1997) Modelling Extremal Events for Insurance and Finance. Berlin: Springer-Verlag.

Engle, R.F. (1982) Autoregressive conditional heteroscedasticity with estimates of the variance of United Kingdom inflation. Econometrica, 50, 987-1008.

Giraitis, L. and Leipus, R. (1990) A functional CLT for nonparametric estimates of spectra and change-point problem for spectral function. Liet. Mat. Rink., 30, 674-697.

Giraitis, L. and Leipus, R. (1992) Testing and estimating in the change-point problem of the spectral function. Liet. Mat. Rink., 32, 20-38.

Giraitis, L., Leipus, R. and Surgailis, D. (1996) The change-point problem for dependent observations. J. Statist. Plann. Inference, 53, 297-310.

Gombay, E., Horváth, L. and Hušková, M. (1996) Estimators and tests for change in variances. Statist. Decisions, 14, 145-159.

He, C. and Teräsvirta, T. (1997) Fourth moment structure of the $\operatorname{GARCH}(p, q)$ process. Working Paper Series in Economics and Finance No. 168, Stockholm School of Economics.

Horváth, L. (1993) The maximum likelihood method for testing changes in the parameters of normal observations. Ann. Statist 21, 671-680.

Horváth, L. and Kokoszka, P. (1997) The effect of long-range dependence on change-point estimators. J. Statist. Plann. Inference, 64, 57-81.

Hsu, D.A. (1977) Tests for variance shift at an unknown time point. Appl. Statist. 26, 279-284.

Inclán, C. and Tiao, G.C. (1994) Use of cumulative sums of squares for retrospective detection of change of variance. J. Amer. Statist. Assoc. 89, 913-923.

Leipus, R. (1994) A posteriori and sequential methods of change-point detection in FARIMA-type time series. In B. Grigelionis, J. Kubilius, H. Pragarauskas and V. Statulevicius (eds), Probability Theory and Mathematical Statistics: Proceedings of the Sixth Vilnius Conference, pp. 485-496. Zeist: VSP/TEV.

Shephard, N. (1996) Statistical aspects of ARCH and stochastic volatility. In D.R. Cox, D.V. Hinkley and O.E. Barndorff-Nielson (eds), Time Series Models in Econometrics, Finance and Other Fields, pp. 1-55. London: Chapman \& Hall.

Received January 1998 and revised September 1998 